\title{
A case study of speculative financial bubbles in the South African stock market 2003-2006
}

\author{
Wei-Xing Zhou ${ }^{\text {a,b,c,d }}$, Didier Sornette ${ }^{e, 1}$ \\ ${ }^{a}$ School of Business, East China University of Science and Technology, Shanghai 200237, \\ China \\ ${ }^{\mathrm{b}}$ School of Science, East China University of Science and Technology, Shanghai 200237, \\ China \\ ${ }^{\mathrm{c}}$ Research Center for Econophysics, East China University of Science and Technology, \\ Shanghai 200237, China \\ ${ }^{\mathrm{d}}$ Research Center of Systems Engineering, East China University of Science and \\ Technology, Shanghai 200237, China \\ ${ }^{\mathrm{e}}$ Chair of Entrepreneurial Risks, Department of Management, Technology, and \\ Economics, ETH Zurich, CH-8032 Zurich, Switzerland
}

\begin{abstract}
We tested 45 indices and common stocks in the South African stock market for the possible existence of a bubble over the period from January 2003 to May 2006. A bubble is defined by a faster-than-exponential acceleration with significant log-periodic oscillations. These two traits are analyzed using different methods. Sensitivity tests shows that the estimated parameters are robust. With the insight of 6 additional month of data since the analysis was performed, we observe that many of the stocks on the South Africa market experienced an abrupt drop mid-June 2006, which is compatible with the predicted $t_{c}$ for several of the stocks, but not all. This suggests that the mini-crash that occurred around mid-June of 2006 was only a partial correction, which has resumed into a renewed bubbly acceleration bound to end some times in 2007, similarly to what happened on the US market from October 1997 to August 1998.
\end{abstract}

Key words: Econophysics; financial bubble; super-exponential acceleration; log-periodicity; power-law singularity; African common stocks

$\overline{1}$ Corresponding author. E-mail address: dsornette@ethz.ch (D. Sornette) http://www.er.ethz.ch/ 


\section{Introduction}

One of the most robust characteristics of humans, which has arguably the most visible imprint in our social affairs, is imitation and herding. Imitation has been documented in psychology and in neuro-sciences as one of the most evolved cognitive process, requiring a developed cortex and sophisticated processing abilities. In short, we learn our basics and how to adapt mostly by imitation all along our life. It seems that imitation has evolved as an evolutionary advantageous trait, and may even have promoted the development of our anomalously large brain (compared with other mammals) [1]. It is actually "rational" to imitate when lacking sufficient time, energy and information to take a decision based only on private information and processing, that is..., most of the time. Imitation, in obvious or subtle forms, is a pervasive activity of humans. In the modern business, economic and financial worlds, the tendency for humans to imitate leads in its strongest form to herding and to crowd effects [2].

Models of cooperative herding and imitation have been built on the notion that imitation leads to positive feedbacks, that is, an action leads to consequences which themselves reinforce the action and so on, leading to virtuous or vicious circles. We have formalized these ideas in the mathematical theory of rational expectation bubbles in the presence of noisy imitative traders. The main idea is to take into account positive feedbacks, due for instance to derivative hedging, portfolio insurance and foremost to imitative trading, as an essential cause for the appearance of nonsustainable bubble regimes. Specifically, the positive feedbacks give rise to power law (i.e., faster than exponential) acceleration of prices. Previous works by us and our co-workers as well as a few other groups suggest that a robust additional feature characterizes the faster-than-exponential growth of prices during bubbles: the existence of accelerating ups and downs, roughly organized according to a geometrically convergence series of characteristic time scales decorating the power law acceleration. Such patterns have been coined "log-periodic power law" (LPPL).

Several mechanisms are known to generate LPPL, suggesting several complementary non-necessarily exclusive explanations. A dynamical explanation [3, 4] consists in taking into account the competition between positive feedback (self-fulfilling sentiment), negative feedbacks (contrariant behavior and fundamental value analysis) and inertia (everything takes time to adjust). The competition between nonlinear trend followers and nonlinear value investors together with inertia between investor decisions and their market impact may lead to nonlinear oscillations approximating log-periodicity [3, 4]. In this case, log-periodicity is nothing but the observable signature of the developing discrete hierarchy of alternating positive and negative feedbacks culminating in the final "rupture," which is the end of the bubble often associated with a crash. Another explanation is based on the natural

hierarchical structure of human groups [5, 6], applied to the network structure of traders [7, 8]. In this class of models, investors in the stock market form a com- 
plex connected hierarchical network and interact with each other "locally" through transfers of information, leading to what we refer broadly as "imitation." Local interactions propagate spontaneously into global cooperation leading to herding behaviors, which result in bubbles. These ingredients, together with the fact that prices reflect the aggregate decisions of investors, is formalized by a rational expectation model of bubbles with imitation between the noise traders [7, 8, 9]. The main consequence of these models is that the dynamics may evolve towards a critical point at a critical time $t_{c}$ corresponding to the most probable end of the bubble. We refer to the book by Sornette [2] for a general introduction, a synthesis of the models and examples of empirical tests and applications.

Mathematically, in its simplest version, the LPPL model is represented by the equation (1) giving the anticipated expected trajectory of the $\log$-price $I(t) \equiv \mathrm{E}[\ln p(t)]$ of a given asset as a function of time, expressed in terms of the distance $\tau=t_{c}-t$ to the critical time $t_{c}$ for bubbles (respectively for antibubbles):

$$
I(t)=A+B \tau^{m}+C \tau^{m} \cos [\omega \ln (\tau)-\phi] .
$$

$A$ is the expected $\log$-price at $t_{c}$ (since $\tau=0$ at $t=t_{c}$, all the other terms are vanishing at $t=t_{c}$ ), $B$ (respectively) controls the amplitude of the power law acceleration (respectively the log-periodic component) of the log-price. The exponent $m$ encodes the structural shape of the acceleration. It is usually found between 0 and 1 , which ensures a finite price at $t_{c}$ together with an asymptotic infinite rate of change close to $t_{c}$. The parameter $\omega$ is the log-periodic angular frequency of the log-periodic oscillations. It should be stressed that $\omega$ is not the inverse of a time scale, but rather it is proportional to the inverse of the logarithm of a scale factor $\lambda$, where $\lambda$ is roughly speaking the ratio of the distances between successive peaks of the log-periodic oscillations. Finally, the phase $\phi$ contains two ingredients: the information on the mechanism of interactions between investors and a rescaling of time [10, 11].

In a nutshell, the LPPL model (1) describes two phenomena: (i) a faster-thanexponential growth (for $0<m<1$ and $B<0$ ) of the expected log-price culminating in a singularity of its slope at the critical time $t_{c}$ and (ii) an accelerating sequence of local rallies and corrections decorating the overall power law acceleration (the "log-periodicity"). The literature cited above contains several derivations of this equation (11) and we refer the reader to them. Basically, the power law part $A+B \tau^{m}$ embodies the effect of collective imitation leading to global herding and a critical behavior characterized mathematically by the singularity at $t_{c}$ (in a way reminiscent to phase transitions in physics, but here in the time domain). The logperiodic component reflects at least two possible effects as mentioned above: (1) an inherent discrete hierarchical structure in the social network of investors and (2) a nonlinear mean reversal behavior of fundamental investing styles. See Ref. [12] for a general review on the symmetry of "discrete scale invariance" at the basis of log-periodicity. 
Table 1

The ticker codes and corresponding numbering of 45 South African stocks (http://www.jse.co.za/) analyzed in the present work.

\begin{tabular}{cccccccccc}
\hline \hline Num & 1 & 2 & 3 & 4 & 5 & 6 & 7 & 8 & 9 \\
Code & J203 & J210 & J257 & J580 & ABL & AGL & AMS & ANG & APN \\
Num & 10 & 11 & 12 & 13 & 14 & 15 & 16 & 17 & 18 \\
Code & ASA & BAW & BIL & BVT & ECO & FSR & GFI & HAR & IMP \\
Num & 19 & 20 & 21 & 22 & 23 & 24 & 25 & 26 & 27 \\
Code & INL & INP & IPL & JDG & KMB & LBT & LGL & MLA & MTN \\
Num & 28 & 29 & 30 & 31 & 32 & 33 & 34 & 35 & 36 \\
Code & NED & NPN & NTC & OML & PIK & PPC & RCH & REM & RMH \\
Num & 37 & 38 & 39 & 40 & 41 & 42 & 43 & 44 & 45 \\
Code & SAB & SAP & SBK & SHF & SLM & SOL & TBS & TKG & WHL \\
\hline \hline
\end{tabular}

The organization of the paper is as follows. The next section 2 tests for the existence of faster-than-exponential acceleration in the price of the 45 stocks used in this study (their ticker code used in the South African stock exchange and the corresponding numbering we use are given in Table 11). It also presents preliminary screening tests on the existence of log-periodicity. Combining these evidences, section 2 finally qualifies 5 assets out of the 45 as exhibiting a significant bubble regime. Then, section 3 presents a detailed analysis of the log-periodic characteristics of these 5 assets, using four different techniques. The estimations of the angular log-frequency $\omega$ 's are found consistent and robust across the four methods. Section 4 presents a sensitivity analysis of the critical time $t_{c}$ and of the angular $\log$-frequency $\omega$ by varying both the starting time as well as the ending time of the interval over which the fits are performed. Section 5 summarizes and concludes with respect to the quality of the forecasts using $t_{c}$ as the most probable time of the corrections associated with the end of the bubbles, using the insight obtained from waiting an additional 6 months since the end of the study in May 2006.

\section{Classification of potential speculative behavior among 45 representative stocks on the Johannesburg Stock Exchange}

One of the most advanced and productive economies in Africa, South Africa is characterized by a developed first world economic infrastructure and an emerging market economy. Its financial market is organized by the Johannesburg Stock Exchange (JSE) (http://www.jse.co.za/), which is the only securities exchange in 
South Africa. It was officially established on 8 November, 1887, and has now more than 400 listed companies [13].

Our study is performed on a subset of 45 companies among the largest companies listed on the JSE, whose ticker codes are given in table1 (for detailed information, see the extended version only available online at http://arXiv.org/abs/physics/0701171). The selection of these 45 companies is representative of the diversification performed by major investment banks and brokerage houses in South Africa, as communicated to us by one of the major brokerage house in South Africa. The daily price series run from the first trading day of January 2003 till May 2006.

\subsection{Is there an acceleration?}

Our first goal is to perform a robust analysis of the the 45 financial time series, to identify those potential candidates for a bubble behavior. There is a large literature on the empirical issue of identifying financial bubbles (see [2, 14, 15, 16, 17, 18, 19, 20, 21, 22] and references therein). A key problem is that bubbles are usually defined as exponentially explosive growth phases, but how can one then distinguish them from the growth of a fundamental valuation process which is also generically expected to follow an exponential growth path? We address this problem by defining a bubble as a faster-than-exponential accelerating price [2, 22], which we refer to as "super-exponential." Being faster than exponential, i.e., the growth rate is itself growing, it is necessarily unsustainable if we assume a standard geometric growth for the underlying economy. A super-exponential path can only be transient, reflecting various positive feedback processes that lead to reinforced growth.

Our goal is thus to contrast a standard exponential growth from a super-exponential growth. The former is characterized by the logarithm of the price of a given asset being linear in time as

$$
\ln [p(t)]=a+b t+\epsilon_{1}
$$

where $\epsilon_{1}$ is a short-hand notation for a random walk component. The parameter $b$ is then the rate of return for continuous compounding of the interests. Expression (2) is nothing but the integrated form of the standard geometrical random walk model with drift. The arguably simplest extension (2) which gives superexponential growth is obtained as

$$
\ln [p(t)]=a+b t+c t^{2}+\epsilon_{2} .
$$

The null hypothesis is $c=0$. If it is rejected with $c>0$ at a sufficiently large significance level, we would conclude that the price process is growing superexponentially, qualifying a bubble regime. Since the residual is not Gaussian and not stationary, we cannot use directly the Wilks' likelihood ratio test or t-test of sig-

nificance for the coefficient $c$. We rather use an approach in the spirit of the Akaike 
Information Criterion (AIC), which amounts to test the two models by comparing $\left[R M S_{1}(i)\right]^{2}$ for model (2) with $\left[R M S_{2}(i)\right]^{2}+2 \sigma^{2}$ for model (3) [23]. The terms $R M S_{1}(i)$ and $R M S_{2}(i)$ are the root-mean-square of the residuals of the fits to the price time series of asset $i$ with model (2) and model (3) respectively. More precisely, the calibration of model (2) determines the best values of the parameters $a$ and $b$ for each stock $i$ and $R M S_{1}(i)$ then provides a measure of the average standard deviation of the residual $\epsilon_{1}$. Similarly, the calibration of model (3) determines the best values of the parameters $a, b$ and $c$ for each stock $i$ and $R M S_{2}(i)$ then provides a measure of the average standard deviation of the residual $\epsilon_{2}$. The term $\sigma^{2}$ is the square of the standard deviation of the noise of the price process. The term $2 \sigma^{2}$ is the cost attributed to model (3) for having one more adjustable parameter compared with model (2). In other words, AIC not only rewards goodness of fit, but also includes a penalty linear in the number of estimated parameters. Then, the AIC qualifies model (3) if $\left[R M S_{2}(i)\right]^{2}+2 \sigma^{2}<\left[R M S_{1}(i)\right]^{2}$. This is equivalent to

$$
\frac{\left[R M S_{1}(i)\right]^{2}-\left[R M S_{2}(i)\right]^{2}}{\left[R M S_{1}(i)\right]^{2}}>\frac{2 \sigma^{2}}{\left[R M S_{1}(i)\right]^{2}} .
$$

The ratio in the 1.h.s. of inequality (4) quantifies the relative goodness of fit of expressions (2) and (3). Since in practice, $\sigma^{2}$ is not known to us, we calculate the relative difference of the improvement of the fits resulting from the additional quadratic term in model (3) in the left-hand-side of (4):

$$
D(i)=\left[R M S_{1}(i)-R M S_{2}(i)\right] / R M S_{1}(i),
$$

for each asset. Then, the larger is the value of $D(i)$, the more probable is the rejection of the null hypothesis and the relevance of the quadratic term qualifying a bubble regime. Here, we put aside the subtle issues [24, 25] stemming from possible spurious regressions resulting from the non-stationarity of the residuals in models (2) and (3), which are left for another work. Fig. 11 gives the 45 values of $D(i)$ for all the 45 stocks.

Fig. 1 suggests the existence of two clusters, characterized by values of the "Relative RMS change" above or below $\approx 25 \%$. We thus disqualify stocks as not being in a bubble regime if they obey at least one of the following criteria:

(1) $D(i) \leqslant 25 \%$,

(2) the quadratic term is positive $(c>0)$,

(3) the overall price has been increasing from the beginning to the end of the period.

This leads us to reject the following 27 stocks as not in a bubble regime from Jan. 2003 till May 2006: 3, 5, 9, 10, 11, 12, 13, 14, 15, 21, 22, 24, 26, 27, 29, 30, 32, 33, $34,35,36,37,38,39,41,44,45$. This selection is in line with visual inspection for all except stock 30, for which the analysis comparing (2) and (3) has not enough power. Indeed, in this case of stock 30, the weak improvement of model (3) may 


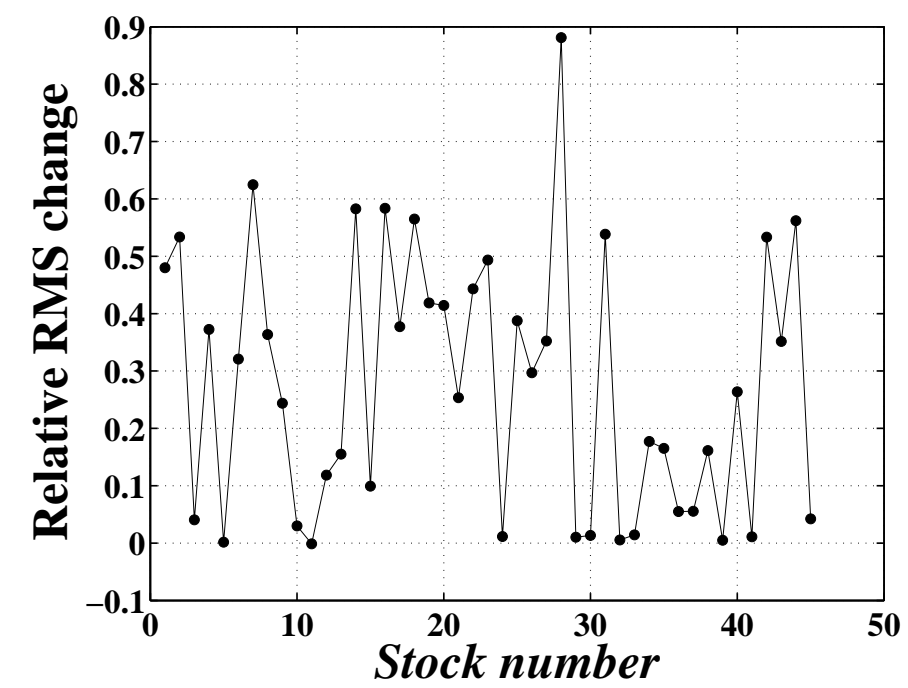

Fig. 1. Relative difference of the improvement of the fits resulting from the additional quadratic term in model (3) in the left-hand-side of (4), as defined by (5).

be attributed to the combination of a large drawup followed by a large drawdown from end-of-2003 to middle-of-2004 followed by an upward acceleration. We thus keep this stock 30 for further analysis.

\subsection{Log-periodic oscillations}

As we just mentioned, the analysis comparing (2) and (3) is not claimed to have universal absolute power: it may have not enough power to reject a stock when it should have been rejected (error of type I or false positive), or it may reject a stock that should be kept in the bubble class (error of type II or false negative) as we argue is perhaps the case of stock 30 .

It is thus useful to examine the results of another test, based on a specification which is well-adapted to test for the presence of super-exponential behavior, in the possible presence of oscillatory intermittent fluctuations. This specification uses the so-called log-periodic formula (1) introduced first in [26, 27] and expanded upon in several subsequent papers (see for instance [2, 28]. We have fitted the 45 stock price series with the log-periodic power-law formula (1) and obtained the key parameters $t_{c}, m$, and $\omega$.

In the above characterization in terms of (2) versus (3), the non-accelerating logprice was characterized essentially by the absence of a significant positive quadratic term $c t^{2}$ in (3). In the present log-periodic power law analysis, a non-accelerating log-price should be qualified by an exponent $m$ close to 1 , while an accelerating log-price corresponds to $m$ small or even negative. We find that this correspondence holds to a large degree, as seen from the following list: $(3,0.86),(5,0.96),(9,0.52)$, 
$(10,0.86),(11,0.84),(12,0.58),(13,0.84),(14,2),(15,0.71),(21,0.27),(22,1.6)$, $(24,0.5),(26,1.6),(27,1.8),(29,0.99),(32,1),(33,0.63),(34,0.41),(35,0.84)$, $(36,1.4),(37,1.3),(38,-2),(39,0.99),(41,0.64),(44,1.4),(45,0.55)$. Here, the first number in each parenthesis stands for the stock number and the second one is the value of the exponent $m$. For the stock prices which have been qualified as nonaccelerating by the method of the previous section, we find values of $m$ typically larger than 0.8 , confirming the classification. The following cases can be considered marginal:

(1) $(9,0.52)$, which has a relative RMS change $D(9) \approx 0.24$ as defined by (5),

(2) $(12,0.58)$, which has a relative RMS change $D(12) \approx 0.12$,

(3) $(21,0.27)$, which has a relative RMS change $D(21) \approx 0.24$,

(4) $(24,0.5)$, which has a relative RMS change $D(24)$ of almost zero, while presenting an intermediate $m$ value,

(5) $(34,0.41)$, which has a relative RMS change $D(34)=0.18$ and the acceleration is more an abrupt change of slope or kink occuring in the first quarter of 2005 , and

(6) $(38,-2)$, which exhibits a log-price which has decreased over most of the time period and which should be rejected.

In addition to the value of the exponent $m$, the presence of log-periodicity has been argued as a signature of a bubble regime [26, 27]. The upper panel of Fig. 2 plots the value of the fitted log-periodic angular frequency $\omega$ for the 45 analyzed stocks. Previous studies on a large number of bubbles mostly on financial indices, bonds and currencies of many developed and emerging countries have shown that the distribution of $\omega$ exhibits several peaks, the prominent one being on the socalled fundamental log-periodic angular frequency $\omega_{1} \approx 6.4 \pm 1.5$ [29], with other peaks on its harmonics $\omega_{n}=n \omega_{1}$. The importance of the high-order harmonics is expected to decrease exponentially [30], but large amplitudes for the second-order and third-order harmonics $\omega_{2}$ and $\omega_{3}$ have been observed to be sometimes very significant [31, 32]. It thus seems difficult to use here a filter based solely on $\omega$, in particular for individual stocks which are necessarily more noisy than aggregate indices.

Ref. [33] has investigated all kinds of scaling series (Bm, fBm, Levy model) to establish the statistical significance level of periodicity and log-periodicity in noisy time series. Ref. [7] also reports extensive tests with GARCH processes to assess the statistical significance level of log-periodicity. Our systematic statistical analysis of the significance level of periodic (as well as log-periodic) signals performed in Ref. [33] shows that the hypothesis, that the observed log-periodicity results from noise, can be rejected at a confidence level higher than $95 \%$ as soon as the the number $N_{\text {osc }}$ of oscillations is 3 or more, for most types of noises. For instance, it has been shown that multiplicative noise on a power law accelerating function leads naturally to stochastic log-periodic oscillations with a most probable number equal to $N_{\text {osc }} \approx 1.5$ [34]. The tests of statistical significance performed in [33] have 


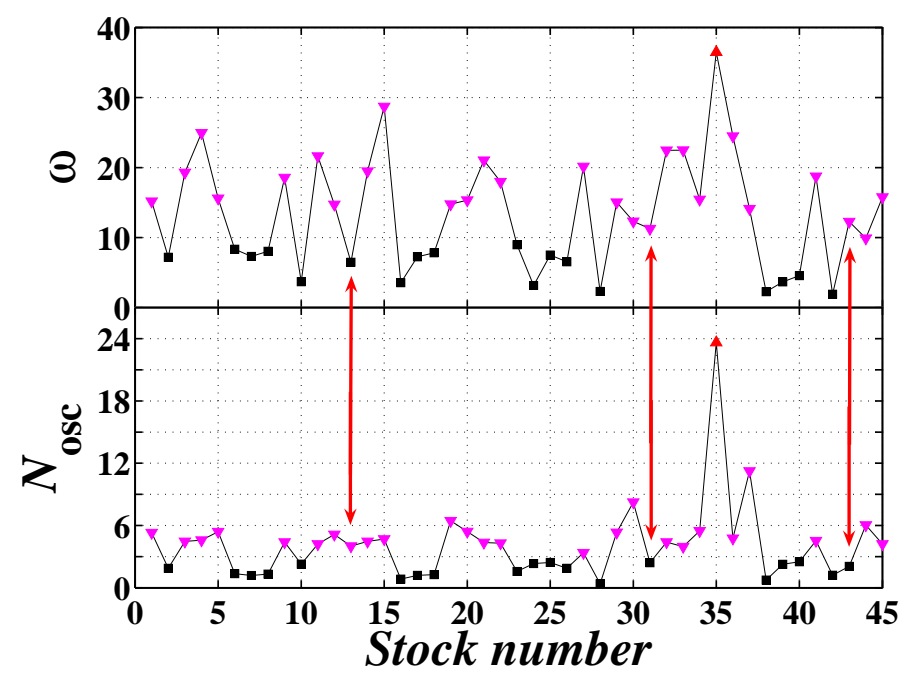

Fig. 2. (Color online). Classification of the 45 analyzed South African stocks with respect to the properties of their log-periodic oscillations. Upper panel: The angular logarithmic frequency $\omega$ for each of the 45 stock indexed with its number $i$. Stocks having $\omega \leqslant 9$ are marked with solid squares, stocks with $9<\omega \leqslant 30$ are indicated by solid downward triangles, and stocks with $\omega \geqslant 30$ are shown with a solid upward triangle. Lower panel: The number of oscillations $N_{\text {osc }}$ over which log-periodicity is found, as a function of the stock number $i$. Stocks having $N_{\text {osc }} \leqslant 3$ are marked with solid squares, those with $3<N_{\text {osc }} \leqslant 10$ are indicated by solid downward triangles, and the stocks with $N_{\text {osc }} \geqslant 10$ are shown with a solid upward triangle.

shown that, for most types of noise, three oscillations are in general sufficient to qualify a genuine oscillatory component. Rigorously, by "genuine", we mean that the probability that the observed oscillatory behavior results from some random noise configuration rather than from some informative signal is below a standard significance level, typically $1-p$ with $p=95 \%$ or $99 \%$. We thus complement the determination of the angular log-frequency $\omega$ by the measure $N_{\text {osc }}$ of log-periodic oscillations in each of the 45 stock prices. Given a LPPL fit and the obtained calibrated parameters $t_{c}$ and $\omega$, the number of oscillations is determined by

$$
N_{\text {osc }}=\frac{\omega}{2 \pi} \ln \left|\frac{t_{c}-t_{\text {first }}}{t_{c}-t_{\text {last }}}\right|
$$

where $\left[t_{\text {first }}, t_{\text {last }}\right]$ is the interval over which the LPPL fitting is performed. The variable $N_{\text {osc }}$ for each of the 45 stocks is shown in the lower panel of Fig. 2.

One can observe that the two measures $\omega$ and $N_{\text {osc }}$ provide consistent results. As shown by the correspondence between the symbols in the upper and lower panel of Fig. 2 for most of the stocks, large $\omega$ 's are associated with a large number of oscillations and vice-versa. There are three exceptions to this observation: $i=13$ (BVT), 31 (OML), and 43 (TBS), outlined in the figure by the arrows. Given the large range of observed $\omega$ 's, we take the number $N_{\text {osc }}$ of LPPL oscillations as the more robust indicator of genuine log-periodicity. This leads to qualify the following 
stocks as exhibiting a significant log-periodicity: 1, 3, 4, 5, 9, 11, 12, 13, 14, 15 , $19,20,21,22,27,29,30,32,33,34,36,37,41,44$, and 45.

Combining this with the results shown in Sec. 2.1, we are left with five stocks which qualify as being in a bubble regime, defined as a significant super-exponential acceleration with the presence of significant log-periodicity: 1 (J203: an index), 4 (J580: an index), 19 (INL), 20 (INP), and 30 (NTC).

In the remaining of this paper, we analyze these five stocks extensively.

\section{Analyzing the log-periodic structure of stocks 1 (J203), 4 (J580), 19 (INL), 20 (INP), and 30 (NTC)}

\subsection{First-order LPPL model}

The fits of the logarithms of the prices of the five stocks 1 (J203), 4 (J580), 19 (INL), 20 (INP), and 30 (NTC) by the log-periodic power law model (1) are shown in Fig. 3 and the parameters are given in Table 2. The small value of the exponent $m$ (except for NTC) confirms a clear super-exponential acceleration. The log-periodic angular frequencies are found to be close to twice (J203, INL, INP, NTC) or four times (J580) the value $\omega_{1} \approx 6.4 \pm 1.5$ of the fundamental log-periodic angular frequency found in many previous studies [22, 29]. The LPPL fits suggested at the time of the fits (end of May 2006) that the bubbles would end either immediately (NTC) or during the second part of the year. We discuss this prediction below.

\section{Table 2}

Parameters of the first-order log-periodic power law model providing the best fits to the the five South African stocks 1 (J203), 4 (J580), 19 (INL), 20 (INP), and 30 (NTC) shown in Fig. 3

\begin{tabular}{ccccccccc}
\hline \hline Stock & $t_{c}$ & $m$ & $\omega$ & $\phi$ & $A$ & $B$ & $10^{3} C$ & $\chi$ \\
\hline J203 (1) & $2006 / 11 / 01$ & 0.27 & 15.3 & 5.41 & 11.3 & -0.336 & 8.73 & 0.036 \\
J580 (4) & $2007 / 11 / 12$ & 0.30 & 24.1 & 4.20 & 12.4 & -0.388 & 4.84 & 0.040 \\
INL (19) & $2006 / 08 / 07$ & 0.37 & 14.1 & 1.89 & 11.3 & -0.159 & 8.08 & 0.072 \\
INP (20) & $2006 / 09 / 19$ & 0.30 & 14.0 & 4.81 & 11.9 & -0.307 & -11.27 & 0.074 \\
NTC (30) & $2006 / 05 / 17$ & 0.60 & 12.8 & 2.21 & 6.9 & -0.014 & -1.91 & 0.054 \\
\hline \hline
\end{tabular}

The detection of log-periodic oscillations, if any, is conveniently performed by removing the global trend of the price of a given stock. One way is to subtract the power law trend from the price and then to analyze the wobbles of the obtained residuals $s(t)$ by an adequate spectral analysis [9]. We shall also use a non- 


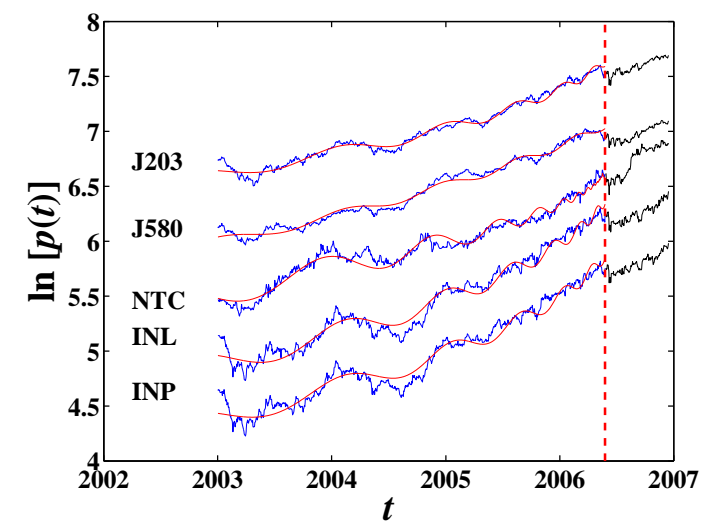

Fig. 3. (Color online). Fits of the first-order LPPL model (1) to the logarithm of the five South African stock prices 1 (J203), 4 (J580), 19 (INL), 20 (INP), and 30 (NTC) from January 2003 to May 2006. The vertical dashed line indicated the end of the fitting interval: May 2006. The rough curves are the historical raw data and extends till Dec. 2006 and the smooth curves are the LPPL fits. The data from June to Dec. 2006 to the right of the vertical dashed line has not been used in the fits. The values of the fit parameters are listed in Table 2. The curves have been shifted vertically for clarity.

parametric approach called the $(H, q)$-analysis [35]. Since log-periodicity corresponds to regular oscillations in the variable $\ln \left(t-t_{c}\right)$, we use a Lomb periodogram analysis which is well-adapted to the uneven sampling of the variable $\ln \left(t-t_{c}\right)$ [36]. The Lomb periodogram analysis is nothing but a spectral analysis based on a least squares fit of sinusoids to data samples, similar to Fourier analysis. The Lomb analysis also allows us to assess the statistical significance level of the extracted $\log$-periodicity [33, 36, 37].

\subsection{Parametric detrending approach}

Following [7, 9], the first method of analysis of the log-periodicity consists in removing the power law trend and then testing for a possible pure log-periodicity without acceleration. We construct the residual $s(t)$ in the following way

$$
s(t)=[I(t)-A] /\left(t-t_{c}\right)^{m} .
$$

This residual $s(t)$ has a nonzero mean $\mu_{s}$ associated with the coefficient $B$ and a given variance $\sigma_{s}^{2}$ (in general different from 1 ). The inset of Fig. 4 plots the standardized residuals $\left[s(t)-\mu_{s}\right] / \sigma_{s}$ as a function of $\ln \tau=\ln \left(t-t_{c}\right)$ for the five stocks 1 (J203), 4 (J580), 19 (INL), 20 (INP), and 30 (NTC). The five Lomb periodograms of these five residuals are presented in Fig. 4. Since $P_{N}(\omega)$ is a normalized Lomb power, $s(t)$ and $\left[s(t)-\mu_{s}\right] / \sigma_{s}$ have identical Lomb periodogram. The angular logperiodic frequencies associated with the highest Lomb peaks are respectively 15.1, $25.1,15.0,15.6$, and 12.5, which are close to the values obtained with the parametric fit with expression (1) listed in Table 2. Their statistical significance is ex- 
tremely high, much larger than $99 \%$, for all possible noise processes, according to the benchmarks developed in [33].

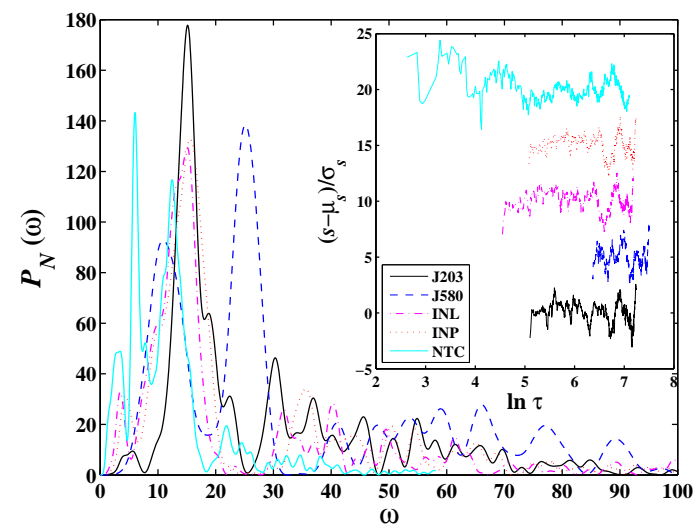

Fig. 4. (Color online). Lomb periodogram analysis of the five standardized residuals $\left[s(t)-\mu_{s}\right] / \sigma_{s}$ shown in the inset, where $s(t)$ is defined by (7), considered as a function of $\ln (\tau)=\ln \left(t-t_{c}\right)$. The curves in the inset have been translated vertically for clarity.

\section{$3.3(H, q)$-analysis}

We have also performed a generalized $q$-analysis, called $(H, q)$-analysis [35, 38], on each of the logarithm of the five price trajectories. The $(H, q)$-analysis is a nonparametric method for characterizing self-similar functions, which generalizes the $q$-analysis [39, 40]. The later is a natural tool for the description of discretely scale invariant fractals. The $(H, q)$-derivative of a function $I(\tau)$ is defined as

$$
D_{q}^{H} I(\tau) \triangleq \frac{I(\tau)-I(q \tau)}{[(1-q) \tau]^{H}}
$$

The special case $H=1$ recovers the standard $q$-derivative, which itself reduces to the standard derivative in the limit $q \rightarrow 1^{-}$. There is no loss of generality by constraining $q$ in the open interval $(0,1)[35]$. The advantage of the $(H, q)$-analysis is that there is no need for detrending, as trends are automatically accounted for by the finite difference and the normalization by the denominator upon a systematic sweeping of the parameter $H$.

We apply the $(H, q)$-analysis to $I(x)=\ln p(t)$, where $p(t)$ is the price of each of the five stocks 1 (J203), 4 (J580), 19 (INL), 20 (INP), and 30 (NTC), as an independent powerful test of log-periodicity. The independent variable is taken to be $\ln \tau$ [35]. The same method has been applied to test for log-periodicity in stock market bubbles and antibubbles [38, 41], in the USA foreign capital inflow bubble ending in early 2001 [42], in the UK real estate bubble [43], in the Chinese stock market antibubble [44], and in the US treasury bond yield antibubble [45].

We scan a $100 \times 50$ rectangular grid in the $(H, q)$ plane, with $H=-0.99: 0.02:$ 
0.99 (from -0.99 to +0.99 with increment 0.02 ) and $q=0.01: 0.02: 0.99$ (from 0.01 to 0.99 with increment 0.02$)$. For each pair of $(H, q)$ values, we calculate the $(H, q)$-derivative (8), on which we perform a Lomb analysis. The highest Lomb peak of the resulting periodogram has height $P_{N}$ and abscissa $\omega$, both $P_{N}$ and $\omega$ being functions of $H$ and $q$. Figure 5 shows the numerically constructed discrete binned bivariate distribution of pairs $\left(\omega, P_{N}\right)$ for $12 \leqslant \omega \leqslant 17$. For values $\omega \leqslant 12$, only one cluster with small values $\omega<4.75$ can be observed: such small values of $\omega$ are associated with only one or at most two oscillations and correspond most probably to the most probable oscillatory structure of multiplicative noise of power law function [34]. Another possible origin of this cluster at $\omega<4.75$ is a residual global trend which has not been completely accounted for by the $(H, q)$-derivative [35, 38].

Most of the Lomb periodograms associated with the points drawn in Fig. 5 have a shape similar to that shown in Fig. 4. Specifically, the average angular log-frequencies determined from this $(H, q)$ analysis are $14.7 \pm 0.3$ for stock "J203", $14.7 \pm 0.1$ for stock "J580", $15.0 \pm 1.3$ for stock "INL", $13.7 \pm 1.5$ for stock "INP", and $15.4 \pm 0.1$ for stock "NTC". In summary, this $(H, q)$-analysis provides even stronger evidence for the existence of log-periodicity than the parametric detrending approach of the previous section 3.2 .

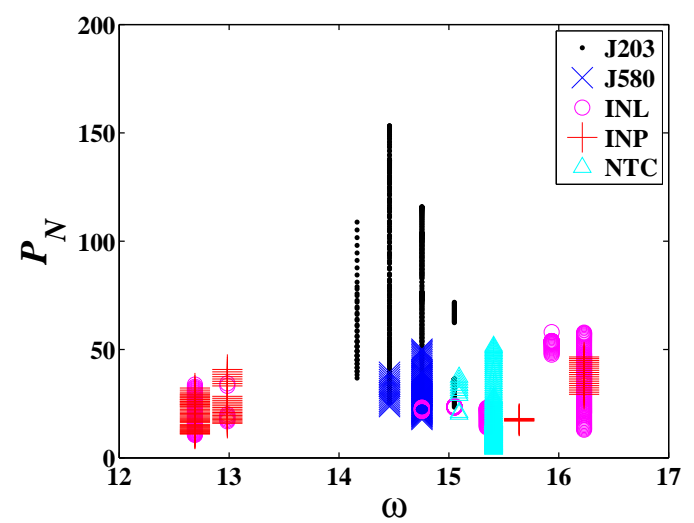

Fig. 5. (Color online). Numerically constructed discrete binned bivariate distribution of pairs $\left(\omega, P_{N}\right)$, defined as the angular log-frequencies and corresponding highest Lomb peaks of the $(H, q)$-derivative of $\ln [p(t)]$, when scanning a $100 \times 50$ rectangular grid in the $(H, q)$ plane, $(H$ from -0.99 to +0.99 with increment 0.02 and $q$ from 0.01 to 0.99 with increment 0.02). Each marker in the figure corresponds to the highest Lomb peak and its associated angular log-frequency in the Lomb periodogram of the $(H, q)$-derivative of $\ln [p(t)]$ for a given pair $(H, q)$.

\subsection{Second-order Weierstrass-type LPPL model}

As already pointed out above, the log-periodic angular frequencies are found to be close to twice (J203, INL, INP, NTC) or four times (J580) the value $\omega_{1} \approx 6.4 \pm 1.5$ 
of the fundamental log-periodic angular frequency found in many previous studies [22, 29]. This suggests that the first-order LPPL formula (1) used until now should be extended to include several harmonics. Indeed, the general mathematical formulation of discrete scale invariance [12, 28] shows that a log-periodic function is expected in general to be represented by a systematic series of log-periodic terms of the form [30]

$$
I(t)=A+B \tau^{m}+\Re\left(\sum_{n=1}^{N} C_{n} \mathrm{e}^{i \psi_{n}} \tau^{-s_{n}}\right),
$$

where $\tau=t_{c}-t$ as before. The main advantage of the high-order Weierstrasstype LPPL models is that they allow to identify the fundamental log-frequency and its harmonics. This family of models have been applied to the case study of many bubbles and antibubbles [32], such as the UK real estate bubble in the last decade [43], the 1975-2001 bubble in the American foreign assets capital inflow [42], the Chinese stock market antibubble since 2001 [44], and the USA treasury bond yield antibubble since 2000 [45].

We adopt the second-order Weierstrass-type LPPL model (9) with $N=2$ to fit the logarithm of the five South African stock prices. The time evolution of the five stocks and the corresponding fits are drawn in Fig. 6 ,

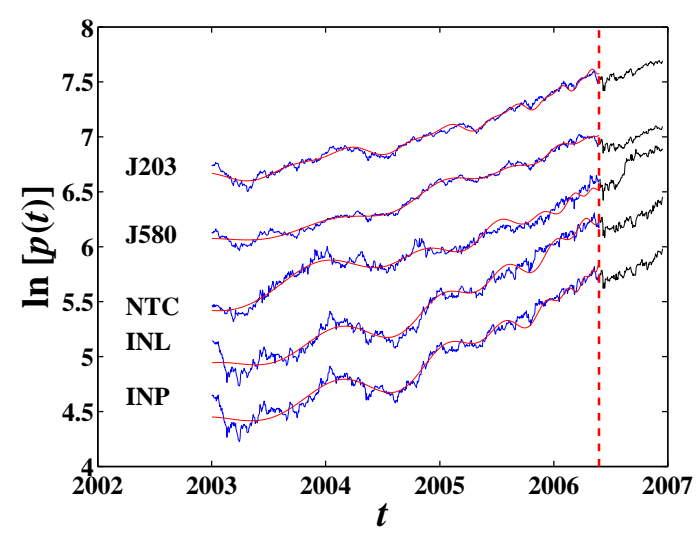

Fig. 6. (Color online). Best fits of the second-order Weierstrass-type LPPL formula (9) with $N=2$ to the logarithm of the five South African stock prices from January 2003 to May 2006. The rough curves are the historical raw data and the smooth curves are the associated LPPL fits. The values of the fit parameters are listed in Table 3 The curves have been shifted vertically for clarity.

The parameters of the five fits using the second-order Weierstrass-type LPPL model are presented in Table 3. We observe that, except from stock J203, the angular logfrequencies lie in the range $6.5 \leq \omega \leq 8.1$, which is consistent with previous results on the fundamental $\log$-frequency $\omega_{1}=6.4 \pm 1.5$ [22, 29]. Specifically, this confirms the coexistence of this fundamental log-frequency together with its harmonics, justifying the interpretation of the large values reported above with the first-order formula (11) as corresponding to the harmonics of $\omega_{1}$. The fact that the 
angular log-frequency $\omega=15.4$ for stock "J203" is close to the second-order harmonic of $\omega_{1}$ is probably associated with a very strong amplitude of the second harmonics, which may hide the existence of $\omega_{1}$. It is also interesting to notice that the absolute values of the linear parameters $C_{1}$ and $C_{2}$ are comparable. In three cases $\left|C_{2}\right|>\left|C_{1}\right|$, indicating that the amplitudes of the second-order harmonic oscillations are considerable, again consistent with our previous interpretation of the results obtained above.

Except for NTC, the exponents $m$ are larger in the second-order LPPL fit than in the first-order case. The critical times $t_{c}$ predicted to be the end of the bubbles are quite robust: they are essentially unchanged for J203 and J580 while they are pushed towards the future by roughly three months for the three other stocks, when going from the first-order to the second-order formula.

Table 3

Parameters of the fits with the second-order Weierstrass-type LPPL model (9) of the five South African stocks

\begin{tabular}{ccccccccccc}
\hline \hline Stock & $t_{c}$ & $m$ & $\omega$ & $\phi_{1}$ & $\phi_{2}$ & $A$ & $B$ & $10^{3} C_{1}$ & $10^{3} C_{2}$ & $\chi$ \\
\hline J203 & $2006 / 11 / 05$ & 0.27 & 15.4 & 5.71 & 3.2 & 11.3 & -0.336 & 8.51 & 4.16 & 0.032 \\
J580 & $2006 / 10 / 27$ & 0.68 & 7.5 & 4.14 & 3.3 & 10.2 & -0.010 & 0.53 & 0.47 & 0.029 \\
INL & $2006 / 11 / 11$ & 0.39 & 8.1 & 4.24 & 2.4 & 11.7 & -0.156 & -4.11 & -6.72 & 0.066 \\
INP & $2006 / 06 / 22$ & 0.61 & 6.5 & 1.58 & 6.2 & 10.7 & -0.021 & 1.39 & 1.87 & 0.062 \\
NTC & $2006 / 08 / 26$ & 0.51 & 7.2 & 5.20 & 5.3 & 7.2 & -0.036 & 1.98 & 2.44 & 0.050 \\
\hline \hline
\end{tabular}

\subsection{Comparison of the different methods}

Let us now compare the estimated angular log-frequencies of the five stock bubbles obtained with the different methods presented above: (i) the fit with the first order LPPL function, (ii) the parametric detrending approach, (iii) the $(H, q)$-analysis, and (iv) the fit with the second-order Weierstrass-type function. The obtained angular log-frequencies are listed in Table 4, The results are self-consistent in the sense that all the bubbles have the same fundamental angular log-frequency $\omega=7.6 \pm 1.9$ and the large values are its higher-order harmonics.

For J203, the four methods give essentially the same value $\omega \approx 15$, which can be interpreted as a very strong second harmonic $2 \omega_{1}$ of the fundamental log-periodic angular log-frequency $\omega_{1} \approx 6.4 \pm 1.5$ found in many previous studies [22, 29].

For J580, the second-order LPPL fit correctly identifies the presence of $\omega_{1}$, and of course its second-order harmonics. The $(H, q)$ analysis identifies the second har- 
Table 4

Comparison of the angular log-frequencies of the five South African stock bubbles estimated by the four different methods.

\begin{tabular}{ccccc}
\hline \hline Stock & LPPL & Detrending & $(H, q)$-analysis & Weierstrass 2 \\
\hline J203 & 15.3 & 15.1 & $14.7 \pm 0.3$ & 15.4 \\
J580 & 24.1 & 25.1 & $14.7 \pm 0.1$ & 7.5 \\
INL & 14.1 & 15.0 & $15.0 \pm 1.3$ & 8.1 \\
INP & 14.0 & 15.6 & $13.7 \pm 1.5$ & 6.5 \\
NTC & 12.8 & 12.5 & $15.4 \pm 0.1$ & 7.2 \\
\hline \hline
\end{tabular}

monics $2 \omega_{1}$ while the two other methods seem to be most sensitive to the fourthorder harmonics.

For the three other stocks, the second-order LPPL fit correctly identifies the presence of $\omega_{1}$, while the three other methods extract its second harmonics $2 \omega_{1}$ as being the dominant contribution, in agreement with the amplitude $C_{2}>C_{1}$ determined in their second-order LPPL fit.

We conclude that genuine log-periodicity exists the price trajectories of these five stocks with high statistical significance, and that the extracted values of the angular log-frequencies are compatible with previous results on other bubbles [22, 29]. The main novelty lies in the importance of the second-order and fourth-order harmonics, which is larger than usual.

\section{Sensitivity analysis of the critical times $t_{c}$}

The determination of the critical time $t_{c}$ is particularly important since it gives the estimated termination time of the bubbles, which can occur approximately two times out of three in the form of a significant correction or a crash. It is noteworthy to stress that a bubble does not end necessarily with a crash as there is a finite probability for a bubble to end with a transition to another regime such as slow deflation or correction [7, 9]. The critical time $t_{c}$ is thus the end of the LPPL bubble and the time at which the crash is most probable, if it ever occurs.

Given its high significance, we have performed a sensitivity analysis of $t_{c}$ for the five stocks with respect to different starting time $t_{\text {first }}$ and ending time $t_{\text {last }}$ of the price time series used in the fitting procedure, following previous works [43, 46, 47]. 


\subsection{The impact of $t_{\text {first }}$}

We first study the impact of $t_{\text {first }}$ on $t_{c}$ and $\omega$ to check the stability of the estimated critical time and the significance of the log-periodic pattern. For each stock, we use the first-order LPPL formula (1) (respectively the second-order LPPL formula (9)) to fit the price time series from $t_{\text {first }}$ to $2006 / 05 / 26$, where $t_{\text {first }}$ ranges from $2003 / 01 / 02$ to $2004 / 12 / 01$ with a spacing of 20 trading days. The results are presented in Fig. 7 (respectively Fig. 8). The overall conclusion is that both $t_{c}$ and $\omega$ are very robust with respect to the choice of the starting time $t_{\text {first }}$ of the fitting interval.
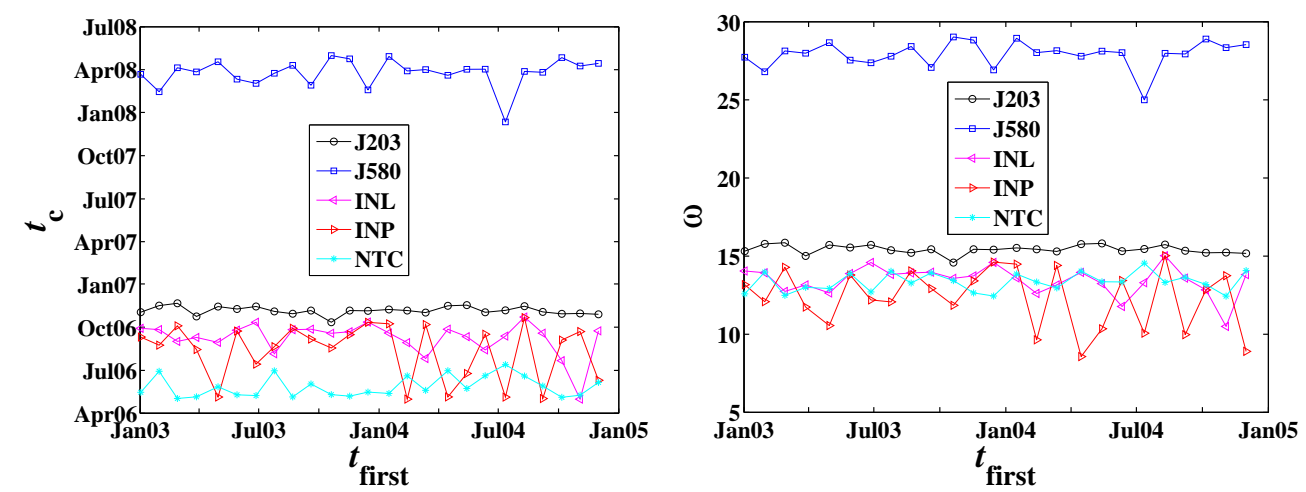

Fig. 7. (Color online). Sensitivity analysis of the estimated critical time $t_{c}$ and the angular $\log$-frequency $\omega$ for the five stocks obtained by varying the last point $t_{\text {first }}$ of the time series up to which the fits using formula (1) are performed.
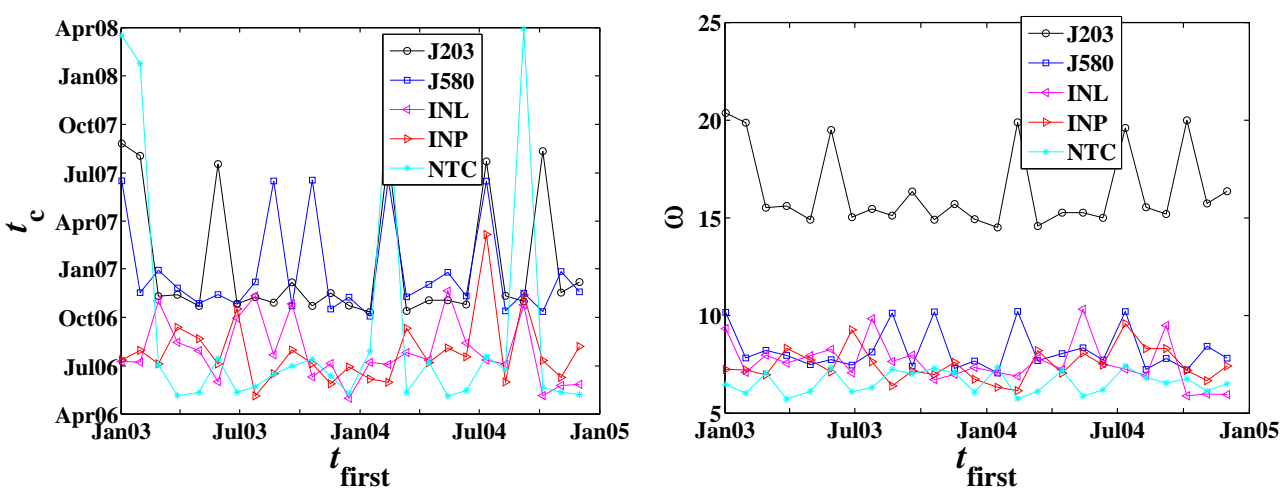

Fig. 8. (Color online). Sensitivity analysis of the estimated critical time $t_{c}$ and the angular $\log$-frequency $\omega$ for the five stocks obtained by varying the last point $t_{\text {first }}$ of the time series up to which the fits using formula (9) with $N=2$ are performed.

\subsection{The impact of $t_{\text {last }}$}

We then study the impact of $t_{\text {last }}$ on $t_{c}$ and $\omega$ to check the stability of the estimated critical time and the significance of the log-periodic pattern. For each stock, we 
use the first-order LPPL formula (1) (respectively the second-order LPPL formula (9)) to fit the price time series from $2003 / 01 / 02$ to $t_{\text {last }}$, where $t_{\text {last }}$ ranges from $2003 / 01 / 02$ to $2004 / 12 / 01$ with a spacing of 20 trading days. The results are presented in Fig. 9 (respectively Fig. 10). Overall, the conclusion is similar than for the dependence on $t_{\text {first }}$, confirming the robustness of the fits and the reliability of our conclusions. The stock NTC is the only one exhibiting a change of regime in the first quarter of 2006, at which the fitted $t_{c}$ jumps from Jan. 2006 to May 2006.
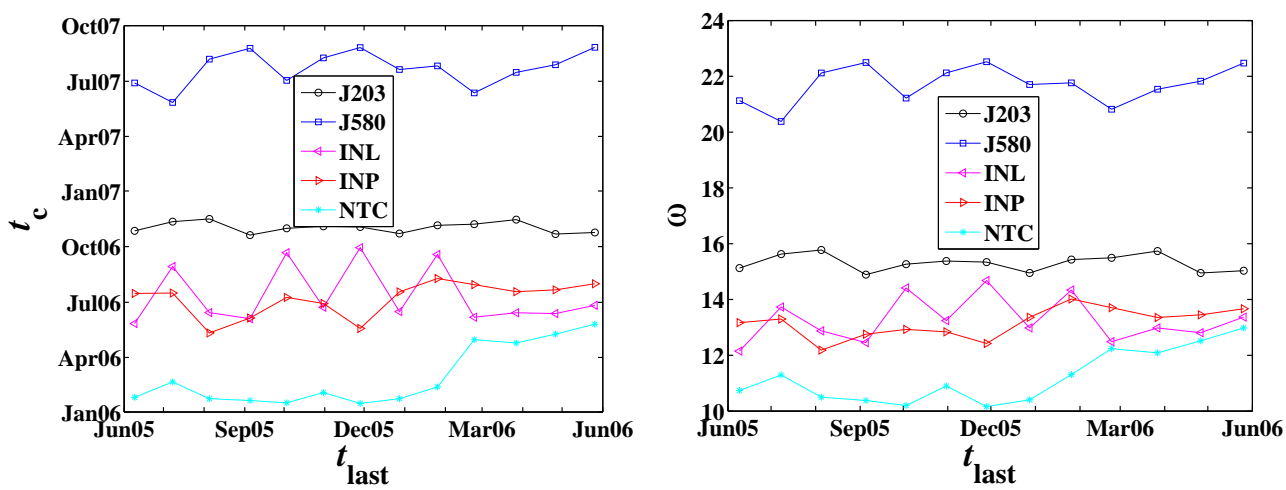

Fig. 9. (Color online). Sensitivity analysis of the estimated critical time $t_{c}$ and the angular $\log$-frequency $\omega$ for the five stocks obtained by varying the last point $t_{\text {last }}$ of the time interval in which the fits using formula (1) are performed.
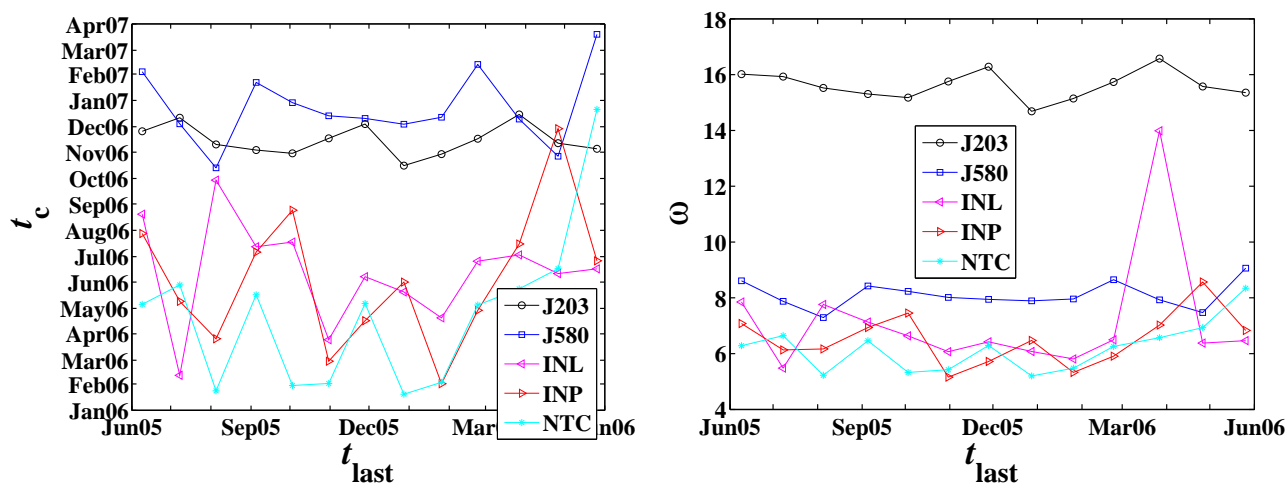

Fig. 10. (Color online). Sensitivity analysis of the estimated critical time $t_{c}$ and the angular $\log$-frequency $\omega$ for the five stocks obtained by varying the last point $t_{\text {last }}$ of the time series up to which the fits using formula (9) with $N=2$ are performed.

\section{Concluding remarks}

In summary, we have identified five stocks (1 (J203), 4 (J580), 19 (INL), 20 (INP), and 30 (NTC)) out of a representative sample of forty five South African stocks, that we qualified as being in a bubble regime defined as a super-exponential growth regime from Jan. 2003 to May 2006 with significant log-periodic oscillations. We 
studied the log-periodic characteristics of these stocks using four different techniques, the parametric fits with the first-order LPPL formula, with the second-order Weierstrass-type model, the parametric detrending method, and the $(H, q)$-analysis. The four techniques give consistent estimations for the value of the fundamental angular log-frequency $\omega_{1}$ in agreement with previous works on many other bubbles in developed and emergent markets, confirming with very high statistical confidence the existence of genuine log-periodicity.

Sensitivity tests of the estimated critical times and of the angular log-frequency by varying the first date and the last date of the stock price time series over which the fits are performed confirm the robustness of the estimated parameters.

This study was performed at the end of May 2006 and we waited another six months before completing this paper to see what were the subsequent evolutions of the five stocks. It turns out that the five selected stocks on the South Africa market experienced an abrupt drop in mid-June 2006, as can be seen from Fig. 3 in which we have shown the subsequent price evolution after May 2006. Quantitatively, the cumulative drawdown (defined as the peak-to-valley relative price variation) measured from the highest price value prior to the end of May 2006 to the price bottom thereafter are respectively: $16.8 \%$ for 1 (J203), $16.6 \%$ for 4 (J580), 20.1\% for 19 (INL), $17.9 \%$ for 20 (INP), and $20.1 \%$ for 30 (NTC). These drawdowns occurred over a time period of less than one month and their amplitudes belong to the 1-percentile of the distribution of drawdowns [48, 49, 50]. The occurrence of these large market price corrections and their timing (mid-June 2006) are compatible with the predicted $t_{c}$ for INL, INP and NTC and to a lesser extend for J203. However, other stocks including J580 give a much large $t_{c}$, some time during 2007, suggesting that the potential for growth in several of these stocks is not exhausted. It is possible that the mini-crash that occurred in mid-June 2006 was only a partial correction, similarly to the Oct. 1997 8\% drop witnessed on the S\&P500 US market which, after being followed by a plateau of three months, resumed in a strong acceleration, to finally end with the real crash in August-September 1998 (see discussion of this sequence in [2]). Finally, we refer to the extended version only available online at http://arXiv.org/abs/physics/0701171, in which the 45 price trajectories for the 45 assets and the fit of the logarithm of their price with the linear model and with the nonlinear model are shown.

\section{Acknowledgments:}

We are grateful to Franco Busetti for providing us the data. This work was partly supported by the National Natural Science Foundation of China (Grant No. 70501011), the Fok Ying Tong Education Foundation (Grant No. 101086), the Program for New Century Excellent Talents in University (Grant No. NCET-07-0288), and the Alfred Kastler Foundation in France. 


\section{References}

[1] R. I. M. Dunbar, The social brain hypothesis, Evol. Anthrop. 6 (1998) 178190.

[2] D. Sornette, Why Stock Markets Crash: Critical Events in Complex Financial Systems, Princeton University Press, Princeton, 2003.

[3] K. Ide, D. Sornette, Oscillatory finite-time singularities in finance, population and rupture, Physica A 307 (2002) 63-106.

[4] D. Sornette, K. Ide, Theory of self-similar oscillatory finite-time singularities, Int. J. Modern Phys. C 14 (2003) 267-275.

[5] W.-X. Zhou, D. Sornette, R. A. Hill, R. I. M. Dunbar, Discrete hierarchical organization of social group sizes, Proc. Royal Soc. B 272 (2005) 439-444.

[6] R. A. Hill, R. A. Bentley, R. Dunbar, Network scaling reveals consistent fractal pattern in hierarchical mammalian societies, Biology Letters (2008) doi:10.1098/rsbl.2008.0393.

[7] A. Johansen, O. Ledoit, D. Sornette, Crashes as critical points, Int. J. Theoret. Appl. Financ. 3 (2000) 219-255.

[8] A. Johansen, D. Sornette, Bubbles and anti-bubbles in Latin-American, Asian and western stock markets: An empirical study, Int. J. Theoret. Appl. Financ. 4 (2001) 853-920.

[9] A. Johansen, D. Sornette, O. Ledoit, Predicting financial crashes using discrete scale invariance, J. Risk 1 (1999) 5-32.

[10] A. Johansen, An alternative view, Quant. Financ. 3 (2003) C6-C7.

[11] D. Sornette, W.-X. Zhou, The US 2000-2003 market descent: Clarifications, Quant. Financ. 3 (2003) C39-C41.

[12] D. Sornette, Discrete scale invariance and complex dimensions, Phys. Rep. 297 (1998) 239-270.

[13] C. Van Zyl, Understanding South African Financial Markets, Schaik Publishers, South Africa, 2006.

[14] J. Hamilton, C. Whiteman, The observable implications of self-fulfilling speculative price, J. Monet. Econ. 16 (1985) 353-373.

[15] G. W. Evans, A test for speculative bubbles in the Sterling-Dollar exchange rate: 1981-84, Am. Econ. Rev. 76 (1986) 621-636.

[16] R. A. Meese, Testing for bubbles in exchange rates: A case of sparkling rates?, J. Polit. Econ. 94 (1986) 345-373.

[17] W. T. Woo, Some evidence of speculative bubbles in the foreign exchange markets, J. Money, Credit, and Banking 19 (1987) 499-514.

[18] C. Camerer, Bubbles and fads in asset prices, J. Econ. Surveys 3 (1989) 3-41.

[19] G. W. Evans, Pitfalls in testing for explosive bubbles in asset prices, Am. Econ. Rev. 81 (1991) 922-930.

[20] M. C. Adam, A. Szafarz, Speculative bubbles and financial markets, Oxford Econ. Papers 44 (1992) 626-640.

[21] R. S. Gurkaynak, Econometric tests of asset price bubbles: Taking stock, FEDS Working Paper (2005) 2005-04.

[22] A. Johansen, D. Sornette, Shocks, crashes and bubbles in financial markets, 
Brussels Economic Review 49, (http://arXiv.org/abs/cond-mat/0210509).

[23] H. Akaike, A new look at the statistical model identification, IEEE Trans. Automat. Contr. 19 (1974) 716-723.

[24] C. Granger, P. Newbold, Spurious regressions in economics,, J. Econometrics 2 (1974) 111-120.

[25] P. C. B. Phillips, Understanding spurious regressions in econometrics, J. Econometrics 33 (1986) 311-340.

[26] D. Sornette, A. Johansen, J.-P. Bouchaud, Stock market crashes, precursors and replicas, J. Phys. I France 6 (1996) 167-175.

[27] J. A. Feigenbaum, P. G. O. Freund, Discrete scale invariance in stock markets before crashes, Int. J. Modern Phys. B 10 (1996) 3737-3745.

[28] D. Sornette, Critical market crashes, Phys. Rep. 378 (2003) 1-98.

[29] A. Johansen, Characterization of large price variations in financial markets, Physica A 324 (2003) 157-166.

[30] S. Gluzman, D. Sornette, Log-periodic route to fractal functions, Phys. Rev. E 65 (2002) 036142.

[31] W.-X. Zhou, D. Sornette, Evidence of intermittent cascades from discrete hierarchical dissipation in turbulence, Physica D 165 (2002) 94-125.

[32] W.-X. Zhou, D. Sornette, Renormalization group analysis of the 2000-2002 anti-bubble in the US S\&P 500 index: Explanation of the hierarchy of five crashes and prediction, Physica A 330 (2003) 584-604.

[33] W.-X. Zhou, D. Sornette, Statistical significance of periodicity and logperiodicity with heavy-tailed correlated noise, Int. J. Modern Phys. C 13 (2002) 137-170.

[34] Y. Huang, A. Johansen, M. W. Lee, H. Saleur, D. Sornette, Artifactual logperiodicity in finite-size data: Relevance for earthquake aftershocks, J. Geophys. Res. 105 (2000) 25451-25471.

[35] W.-X. Zhou, D. Sornette, Generalized $q$-analysis of log-periodicity: Applications to critical ruptures, Phys. Rev. E 66 (2002) 046111.

[36] W. Press, S. Teukolsky, W. Vetterling, B. Flannery, Numerical Recipes in FORTRAN: The Art of Scientific Computing, Cambridge University Press, Cambridge, 1996.

[37] H.-C. G. van Bothmer, C. Meister, Predictingcritical crashes? A new restriction for the free variables, Physica A 320 (2003) 539-547.

[38] W.-X. Zhou, D. Sornette, Nonparametric analyses of log-periodic precursors to financial crashes, Int. J. Modern Phys. C 14 (2003) 1107-1125.

[39] A. Erzan, Finite $q$-differences and the discrete renormalization group, Phys. Lett. A 225 (1997) 235-238.

[40] A. Erzan, J.-P. Eckmann, $q$-analysis of fractal sets, Phys. Rev. Lett. 78 (1997) 3245-3248.

[41] D. Sornette, W.-X. Zhou, The US 2000-2002 market descent: How much longer and deeper?, Quant. Financ. 2 (2002) 468-481.

[42] D. Sornette, W.-X. Zhou, Evidence of fueling of the 2000 new economy bubble by foreign capital inflow: Implications for the future of the US economy and its stock market, Physica A 332 (2004) 412-440. 
[43] W.-X. Zhou, D. Sornette, 2000-2003 real estate bubble in the UK but not in the USA, Physica A 329 (2003) 249-263.

[44] W.-X. Zhou, D. Sornette, Antibubble and prediction of China's stock market and real-estate, Physica A 337 (2004) 243-268.

[45] W.-X. Zhou, D. Sornette, Causal slaving of the U.S. treasury bond yield antibubble by the stock market antibubble of August 2000, Physica A 337 (2004) 586-608.

[46] W.-X. Zhou, D. Sornette, Testing the stability of the 2000 US stock market "antibubble", Physica A 348 (2005) 428-452.

[47] W.-X. Zhou, D. Sornette, Fundamental factors versus herding in the 20002005 US stock market and prediction, Physica A 360 (2006) 459-482.

[48] A. Johansen, D. Sornette, Stock market crashes are outliers, Eur. Phys. J. B 1 (1998) 141-143.

[49] A. Johansen, D. Sornette, Large stock market price drawdowns are outliers, J. Risk 4 (2) (2001) 69-110.

[50] D. Sornette, A. Johansen, Significance of log-periodic precursors to financial crashes, Quant. Financ. 1 (2001) 452-471. 


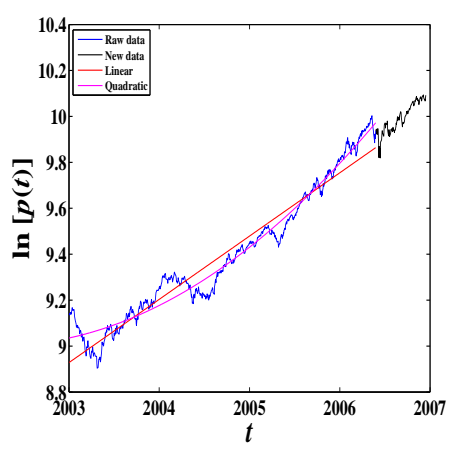

(a) Stock No. 1: J203

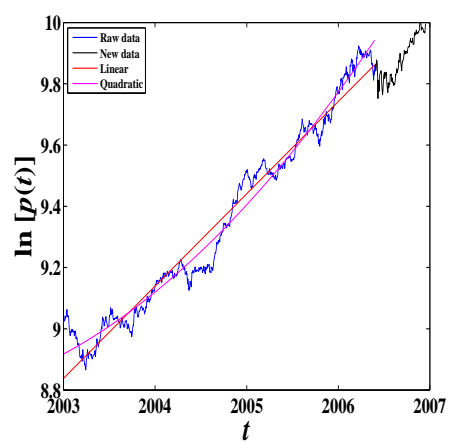

(d) Stock No. 4: J580

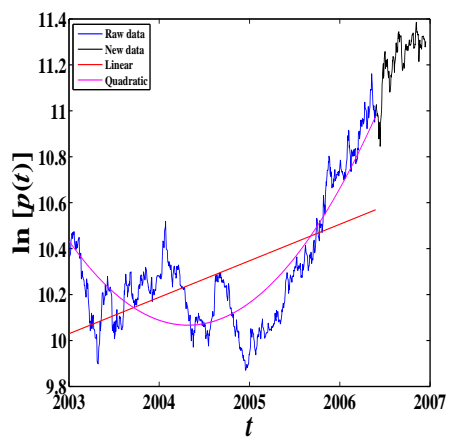

(g) Stock No. 7: AMS

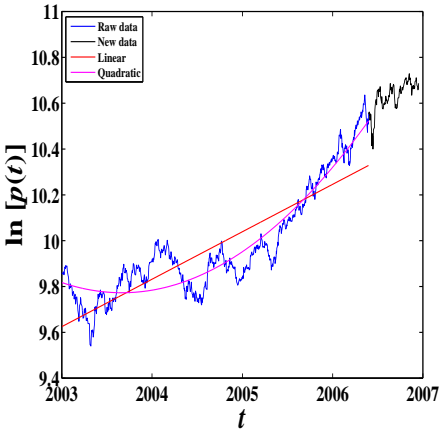

(b) Stock No. 2: J210

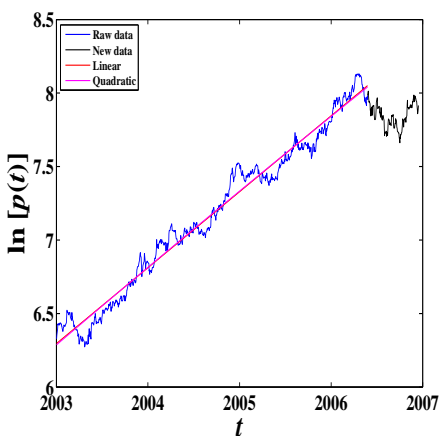

(e) Stock No. 5: ABL

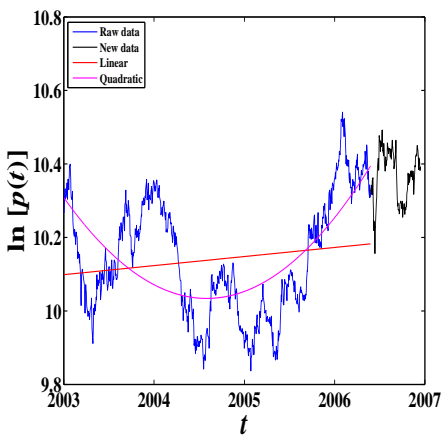

(h) Stock No. 8: ANG

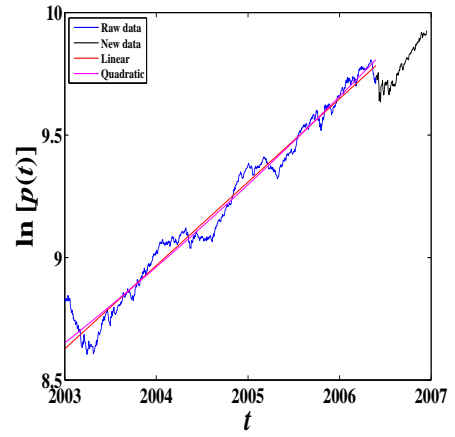

(c) Stock No. 3: J257

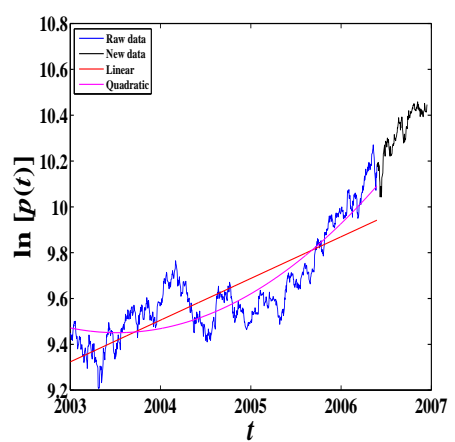

(f) Stock No. 6: AGL

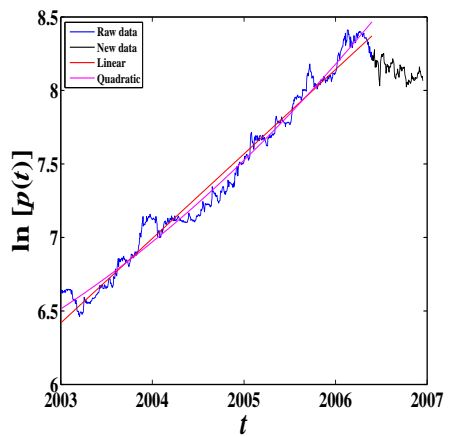

(i) Stock No. 9: APN

Fig. 11. Linear fits and quadratic fits of the prices of stocks from No. 1 to No. 9. 
Table 5. The 45 financial time series analyzed. Rows in red are indices.

\begin{tabular}{|c|c|c|c|}
\hline Ticker & Company name & Major Industry & Sub Industry \\
\hline $\mathrm{J} 203$ & JH-OVER & FTSE/JSE Africa All Share & \\
\hline $\mathrm{J} 210$ & JSE-RESI & FTSE/JSE Africa Resource 20 & \\
\hline $\mathrm{J} 257$ & JH-ASIN & FTSE/JSE Africa All Share Industrials & \\
\hline J580 & JSE-FINA & FTSE/JSE Africa All Share Financial & \\
\hline $\mathrm{ABL}$ & African Bank Investments Ltd & Financial & Commercial banks \\
\hline AGL & Anglo American Plc & Metal producers \& Products manufacturers & Miscellaneous metal producers \\
\hline AMS & Anglo Platinum Ltd & Metal producers \& Products manufacturers & Miscellaneous metal producers \\
\hline ANG & Anglogold Ashanti Ltd & Metal producers \& Products manufacturers & Gold producers \\
\hline APN & Aspen Pharmacare Holdings Ltd & Drugs, cosmetics \& health care & Diversified drugs, cosmetics, $\&$ health care \\
\hline ASA & ABSA Group Ltd & Financial & Commercial banks \\
\hline BAW & Barloworld Limited & Diversified & General diversified \\
\hline BIL & BHP Billiton Plc & Metal producers \& Products manufacturers & Diversified metal producers \\
\hline BVT & Bidvest Group Ltd & Diversified & General diversified \\
\hline ECO & Edgars Consolidated Stores Ltd & Retailers & Apparel store chains \\
\hline FSR & FirstRand Ltd & Financial & Commercial banks \\
\hline
\end{tabular}


Table 6. The 45 financial time series analyzed (Continued).

\begin{tabular}{llll}
\hline \hline Ticker & Company name & Major Industry & Sub Industry \\
\hline GFI & Gold Fields Ltd & Metal producers \& Products manufacturers & Gold producers \\
HAR & Harmony Gold Mining Company Ltd & Metal producers \& Products manufacturers & Gold producers \\
IMP & Impala Platinum Holdings Ltd & Metal producers \& Products manufacturers & Miscellaneous metal producers \\
INL & Investec Ltd & Financial & Commercial banks \\
INP & Investec Ltd & Financial & Commercial banks \\
IPL & Imperial Holdings Ltd & Transportation & Other transportation \\
JDG & JD Group Ltd & Retailers & Miscellaneous retailers \\
KMB & Kumba Resources Ltd & Metal producers \& Products manufacturers & Diversified metal producers \\
LBT & Liberty International Plc & Financial & Land and real estate \\
LGL & Liberty Group Ltd & Financial & Insurance companies \\
MLA & Mittal Steel South Africa Ltd & Metal producers \& Products manufacturers & Diversified metal producers \\
MTN & MTN Group Ltd & Utilities & Telecommunications \\
NED & Nedbank Group Ltd & Financial & Commercial banks \\
NPN & Naspers Ltd & Recreation & Radio \& TV broadcasts \\
NTC & Network Healthcare Holdings Ltd & Miscellaneous & Medical services \\
\hline \hline
\end{tabular}


Table 7. The 45 financial time series analyzed (Continued)

\begin{tabular}{llll}
\hline \hline Ticker & Company name & Major Industry & Sub Industry \\
\hline OML & Old Mutual plc & Financial & Insurance companies \\
PIK & Pick n Pay Stores Ltd & Retailers & Miscellaneous retailers \\
PPC & Pretoria Portland Cement Company Ltd & Construction & Cement producers \\
RCH & Richemont Securities Ag & Clothing \& Footware & \\
REM & Remgro Ltd & Diversified & General diversified \\
RMH & RMB Holdings Ltd & Financial & Other financial services \\
SAB & SABMiller plc & Food \& beverages & Brewers \\
SAP & Sappi Ltd & Paper & Diversified paper \\
SBK & Standard Bank Group Ltd & Financial & Commercial banks \\
SHF & Steinhoff International Holdings Ltd & Miscellaneous & Furnishings \\
SLM & Sanlam Ltd & Financial & Insurance companies \\
SOL & Sasol Ltd & Chemicals & Diversified chemical mfrs. \\
TBS & Tiger Brands Ltd & Diversified & General diversified \\
TKG & Telkom SA Ltd & Utilities & Telecommunications \\
WHL & Woolworths Holdings Ltd & Retailers & Miscellaneous retailers \\
\hline \hline
\end{tabular}




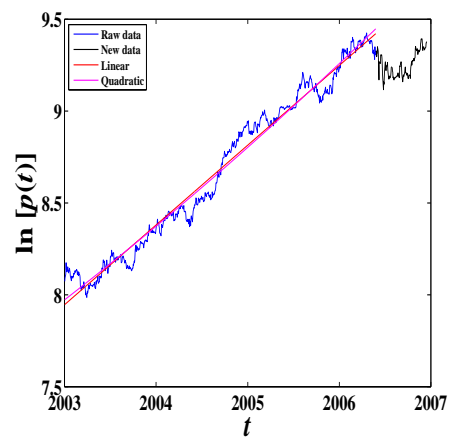

(a) Stock No.10: ASA

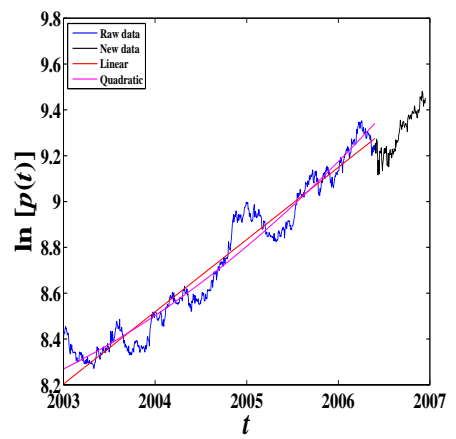

(d) Stock No.13: BVT

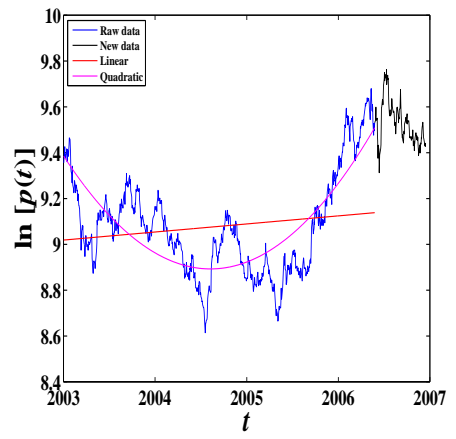

(g) Stock No.16: GFI

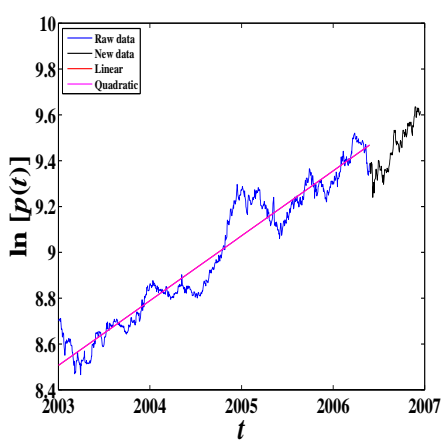

(b) Stock No.11: BAW

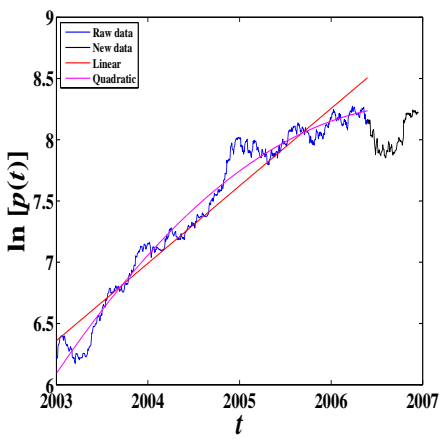

(e) Stock No.14: ECO

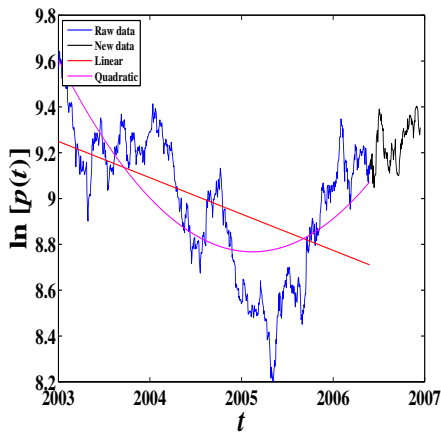

(h) Stock No.17: HAR

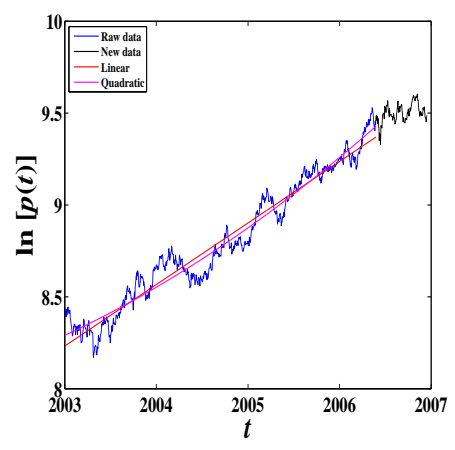

(c) Stock No.12: BIL

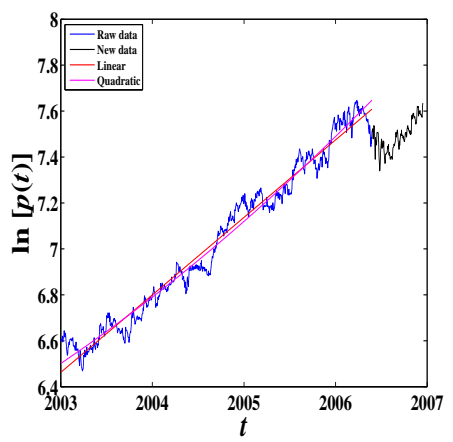

(f) Stock No.15: FSR

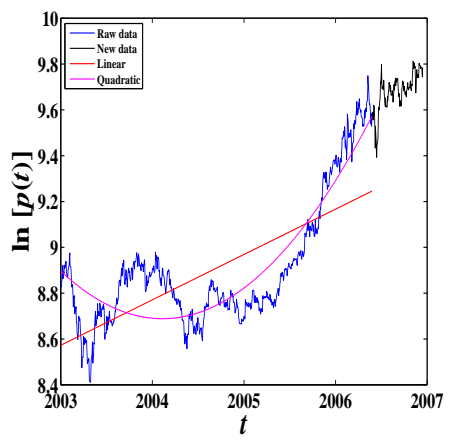

(i) Stock No.18: IMP

Fig. 12. Linear fits and quadratic fits of the prices of stocks from No.10 to No.18. 


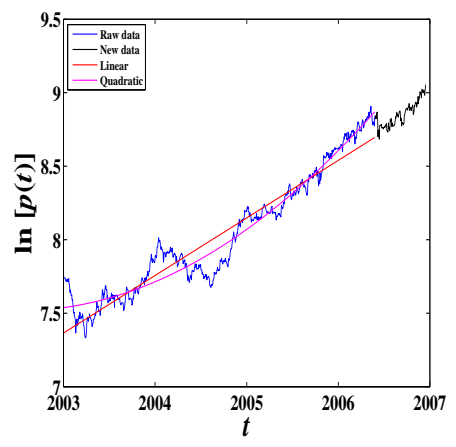

(a) Stock No.19: INL

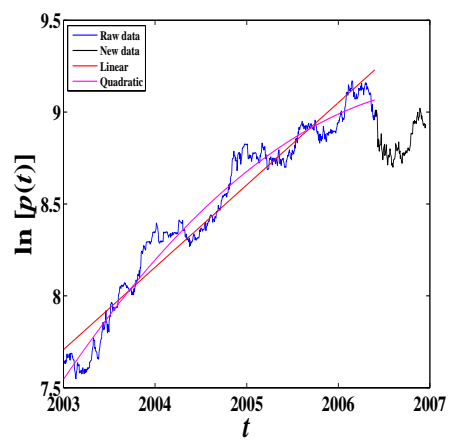

(d) Stock No.22: JDG

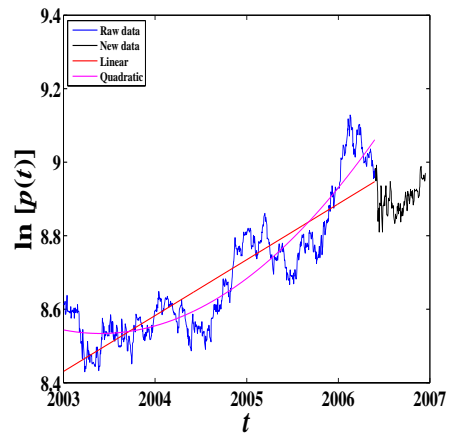

(g) Stock No.25: LGL

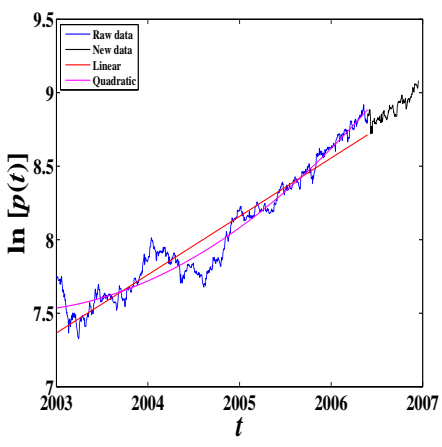

(b) Stock No.20: INP

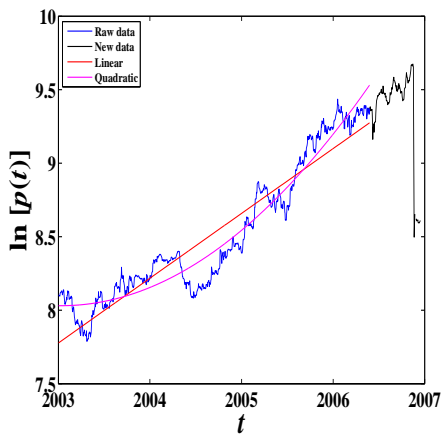

(e) Stock No.23: KMB

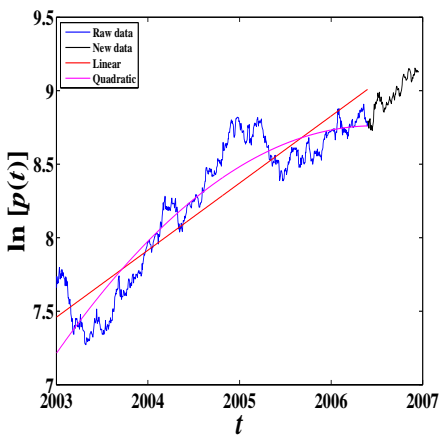

(h) Stock No.26: MLA

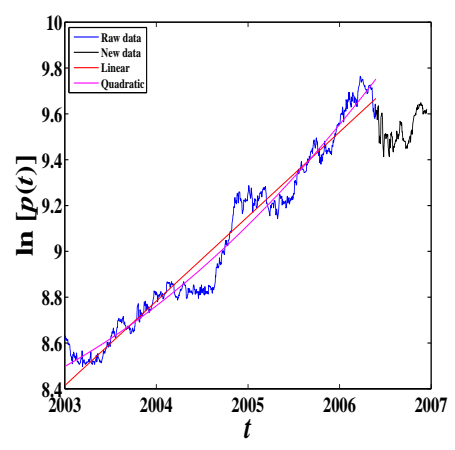

(c) Stock No.21: IPL

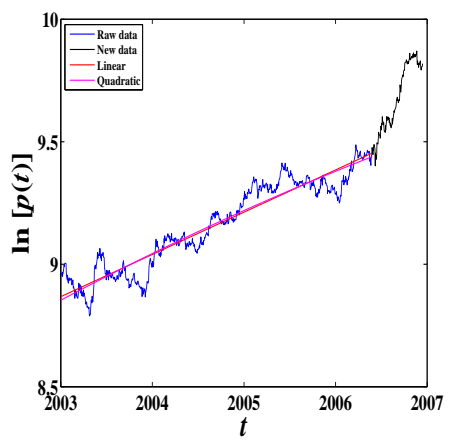

(f) Stock No.24: LBT

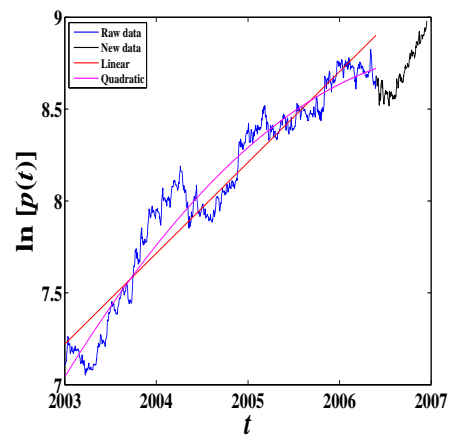

(i) Stock No.27: MTN

Fig. 13. Linear fits and quadratic fits of the prices of stocks from No.19 to No.27. 


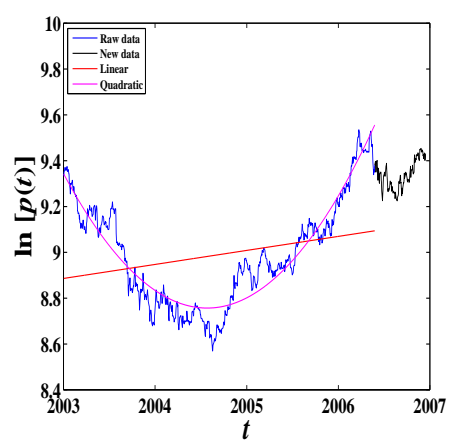

(a) Stock No.28: NED

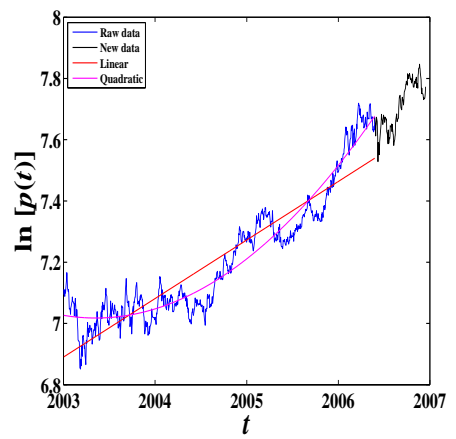

(d) Stock No.31: OML

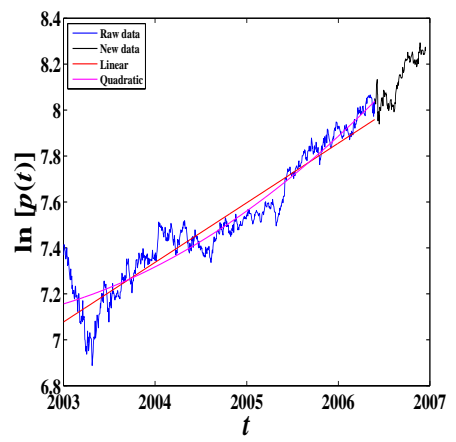

(g) Stock No.34: $\mathrm{RCH}$

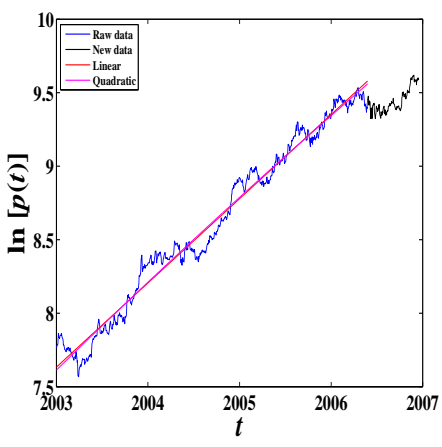

(b) Stock No.29: NPN

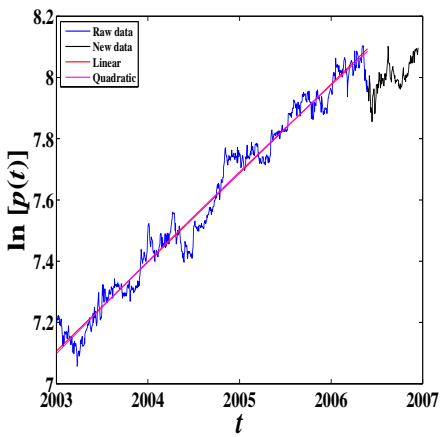

(e) Stock No.32: PIK

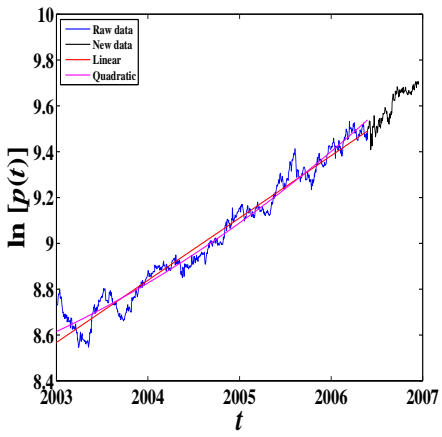

(h) Stock No.35: REM

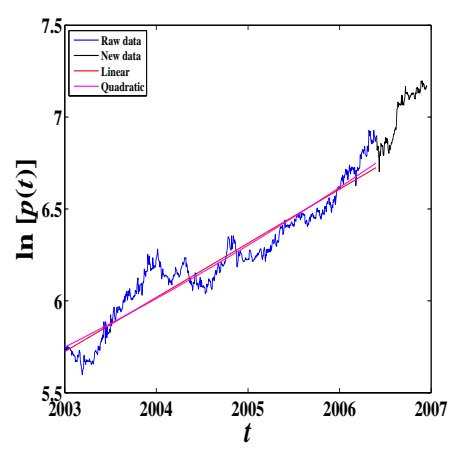

(c) Stock No.30: NTC

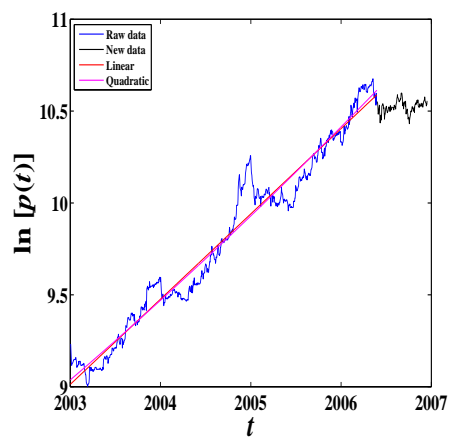

(f) Stock No.33: PPC

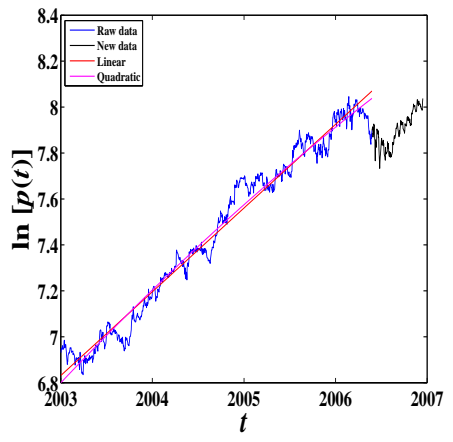

(i) Stock No.36: RMH

Fig. 14. Linear fits and quadratic fits of the prices of stocks from No.28 to No.36. 


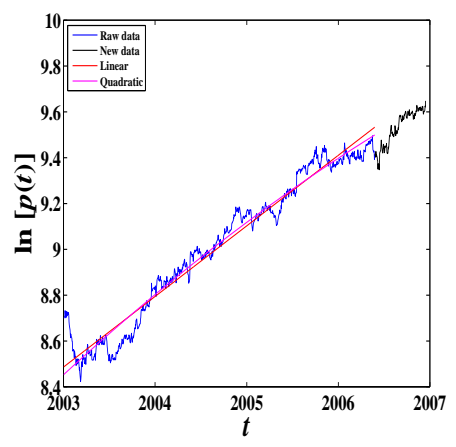

(a) Stock No.37: SAB

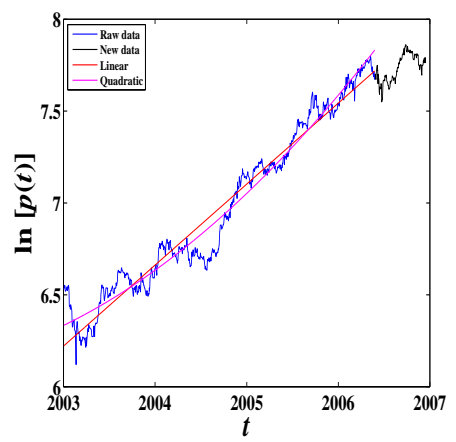

(d) Stock No.40: SHF

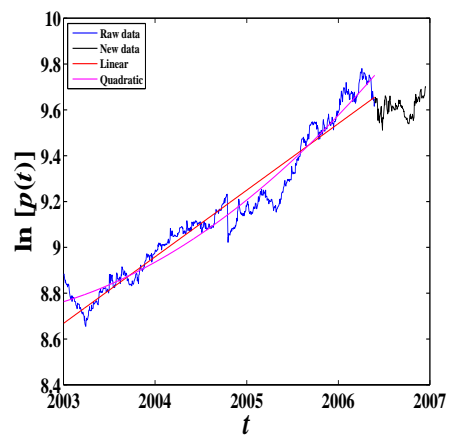

(g) Stock No.43: TBS

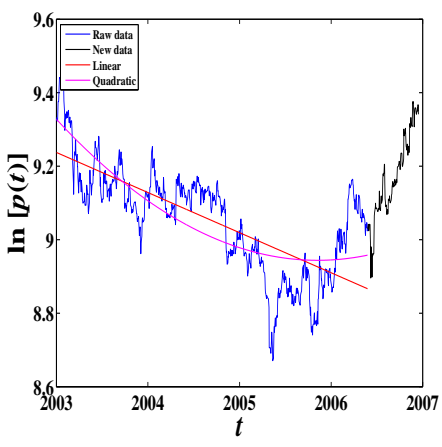

(b) Stock No.38: SAP

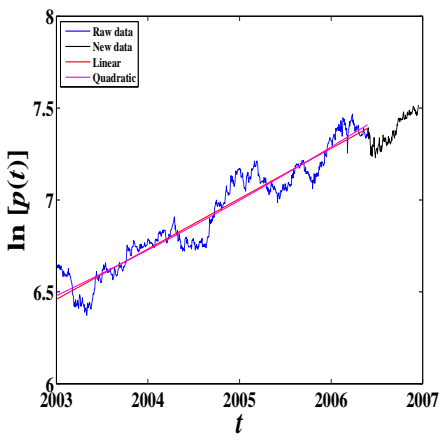

(e) Stock No.41: SLM

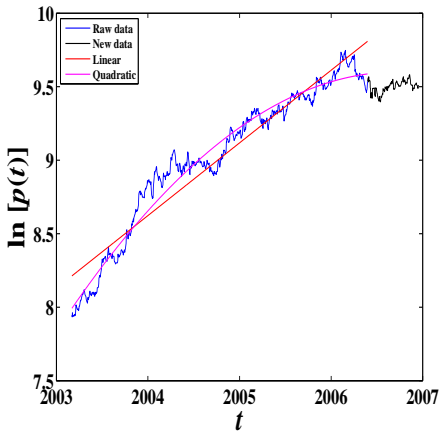

(h) Stock No.44: TKG

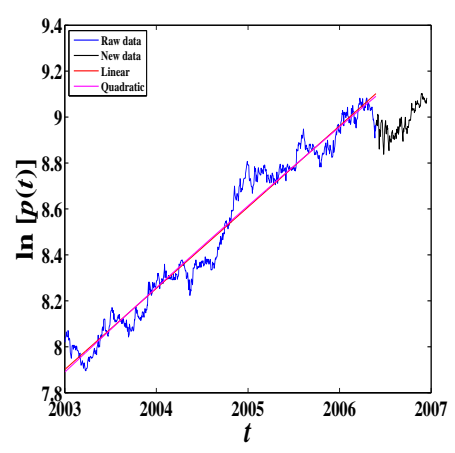

(c) Stock No.39: SBK

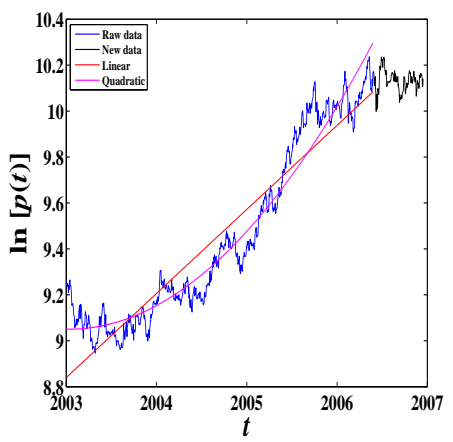

(f) Stock No.42: SOL

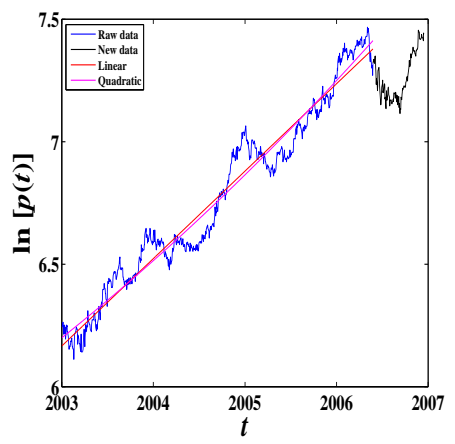

(i) Stock No.45: WHL

Fig. 15. Linear fits and quadratic fits of the prices of stocks from No.37 to No.45. 


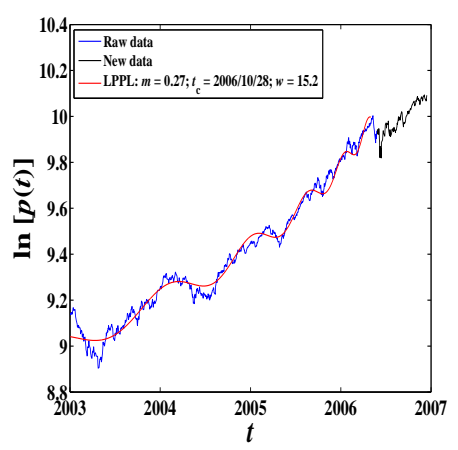

(a) Stock No. 1: J203

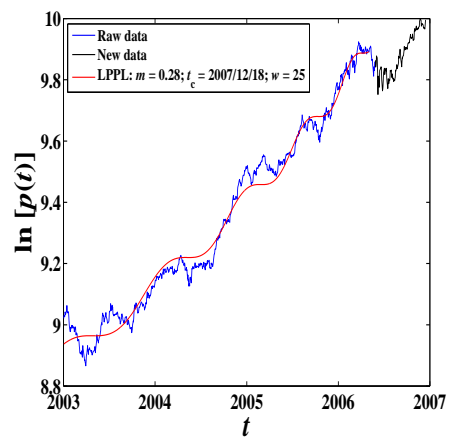

(d) Stock No. 4: J580

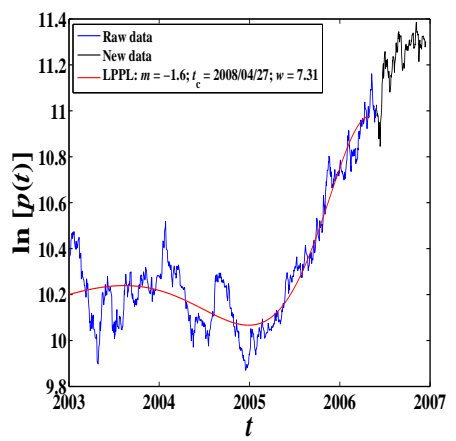

(g) Stock No. 7: AMS

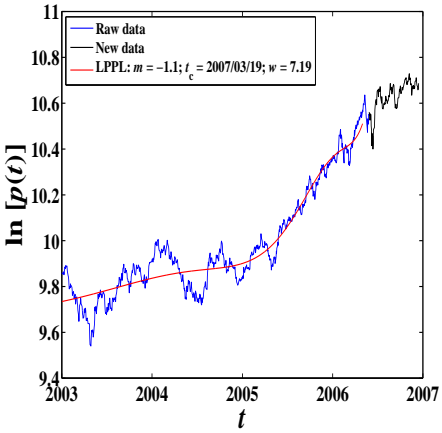

(b) Stock No. 2: J210

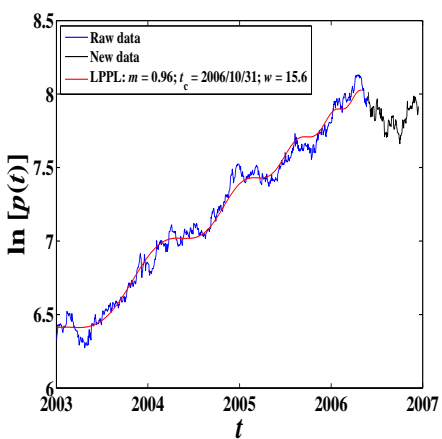

(e) Stock No. 5: ABL

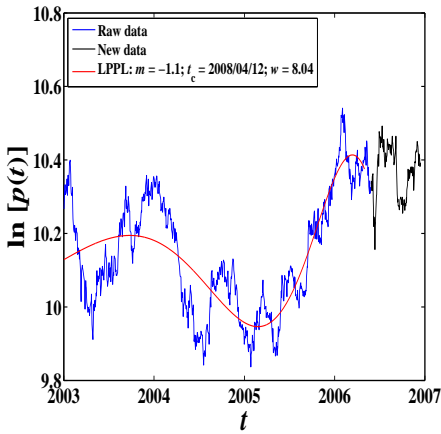

(h) Stock No. 8: ANG

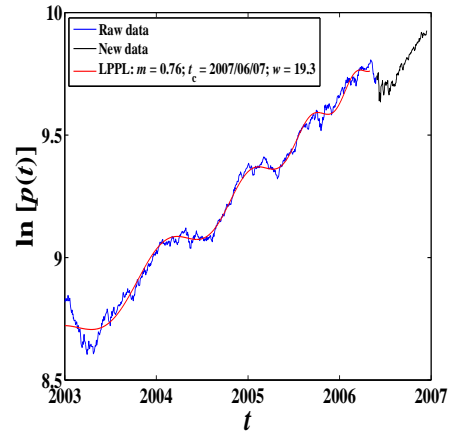

(c) Stock No. 3: J257

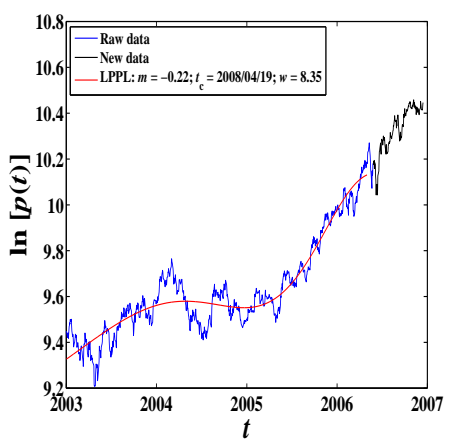

(f) Stock No. 6: AGL

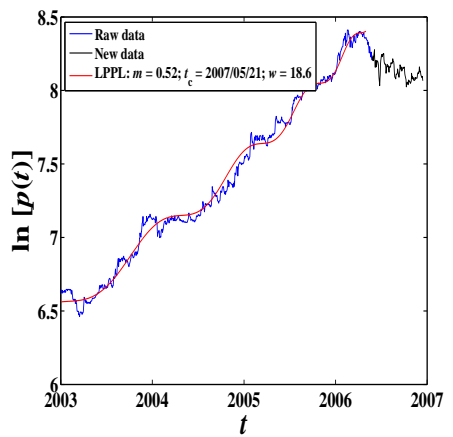

(i) Stock No. 9: APN

Fig. 16. Log-periodic power-law fits of the prices of stocks from No. 1 to No. 9. 


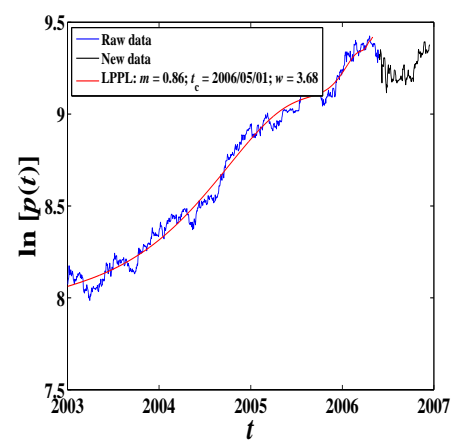

(a) Stock No.10: ASA

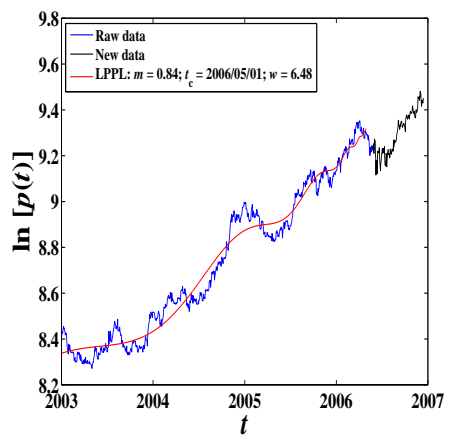

(d) Stock No.13: BVT

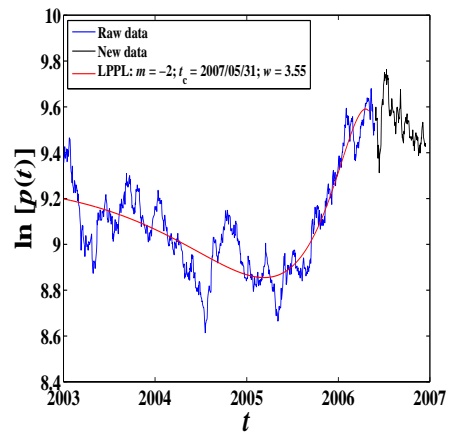

(g) Stock No.16: GFI

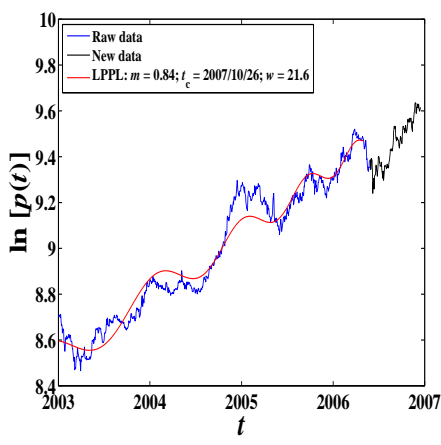

(b) Stock No.11: BAW

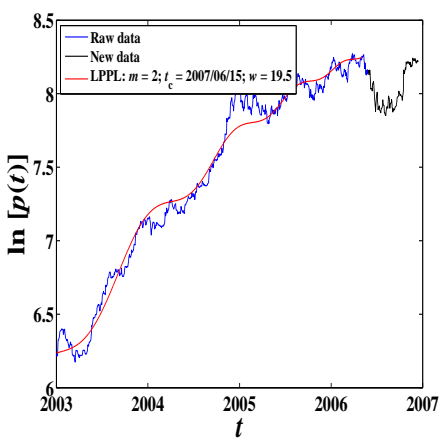

(e) Stock No.14: ECO

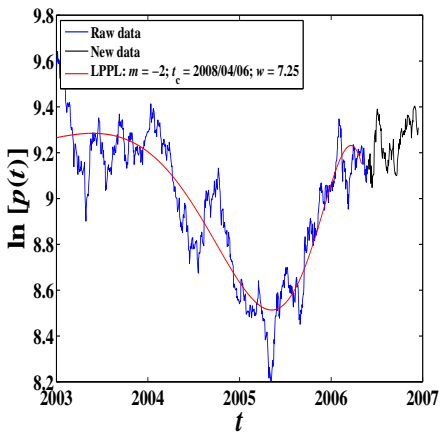

(h) Stock No.17: HAR

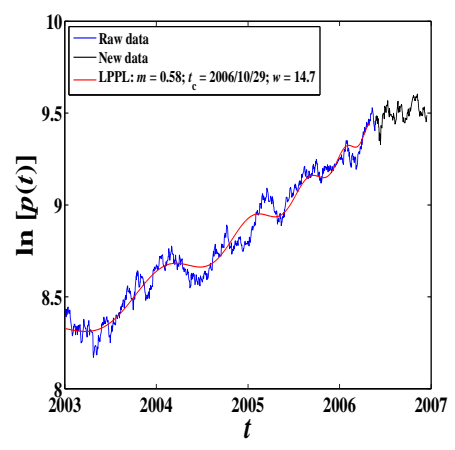

(c) Stock No.12: BIL

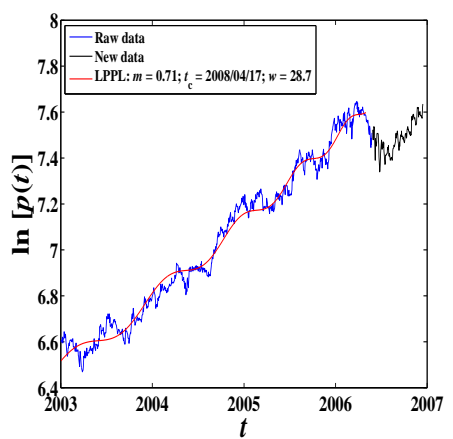

(f) Stock No.15: FSR

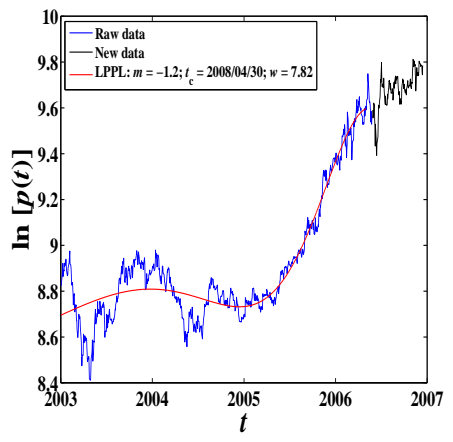

(i) Stock No.18: IMP

Fig. 17. Log-periodic power-law fits of the prices of stocks from No.10 to No.18. 


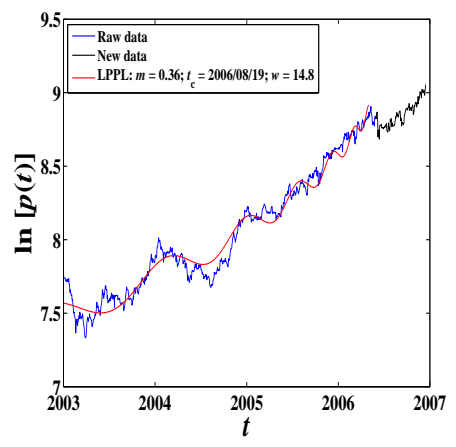

(a) Stock No.19: INL

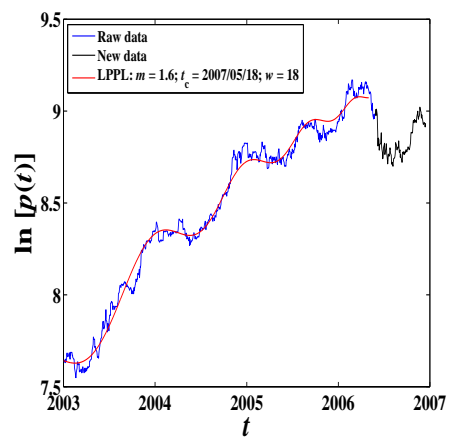

(d) Stock No.22: JDG

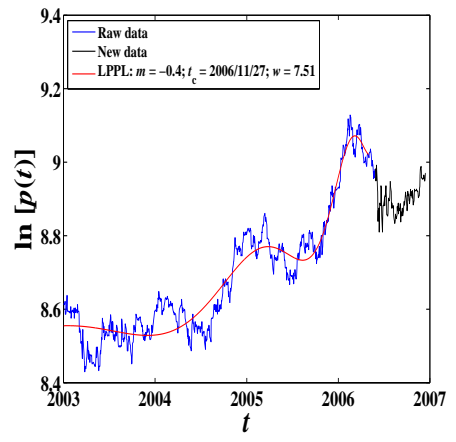

(g) Stock No.25: LGL

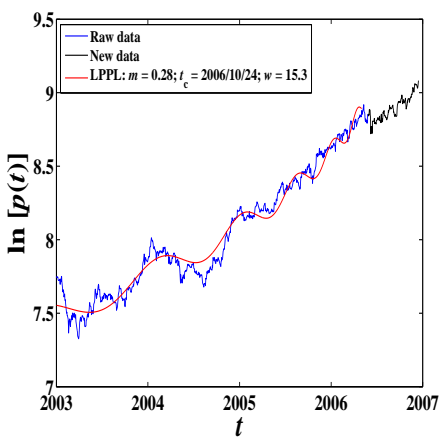

(b) Stock No.20: INP

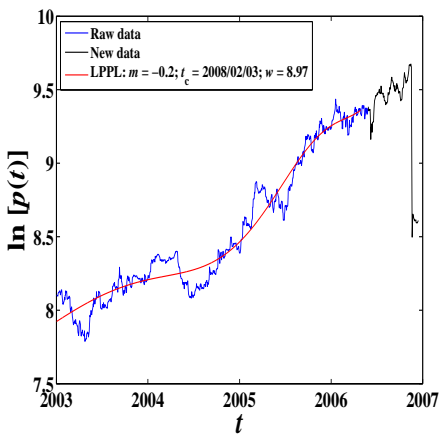

(e) Stock No.23: KMB

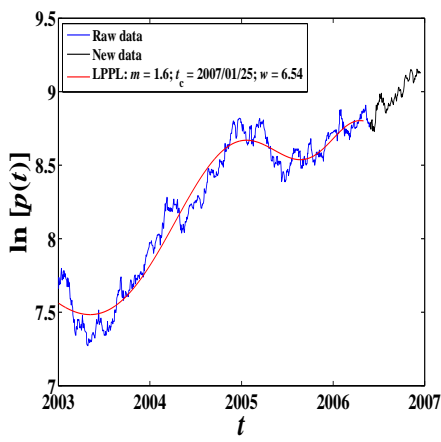

(h) Stock No.26: MLA

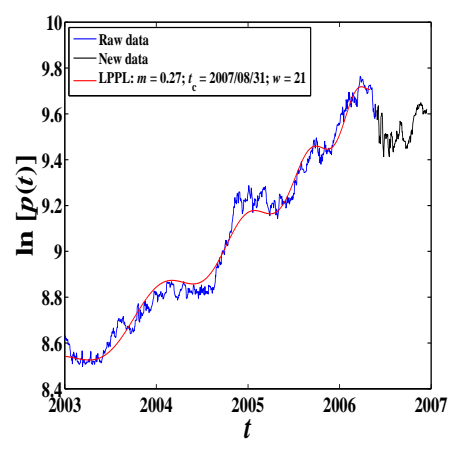

(c) Stock No.21: IPL

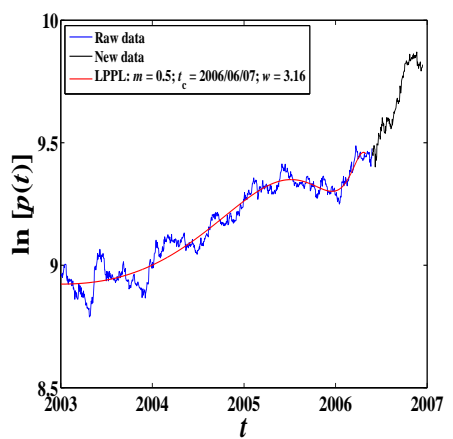

(f) Stock No.24: LBT

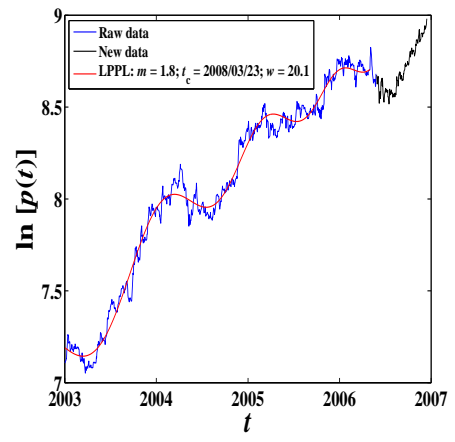

(i) Stock No.27: MTN

Fig. 18. Log-periodic power-law fits of the prices of stocks from No.19 to No.27. 


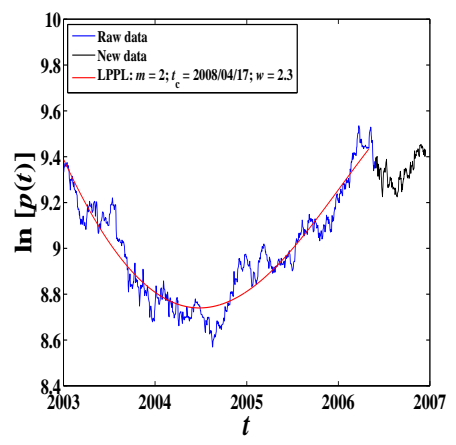

(a) Stock No.28: NED

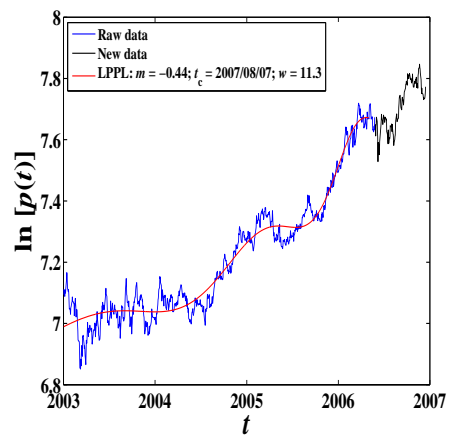

(d) Stock No.31: OML

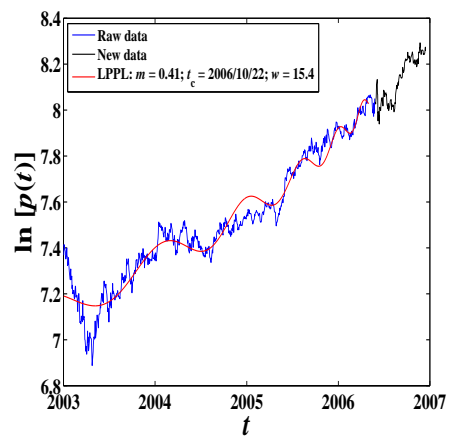

(g) Stock No.34: RCH

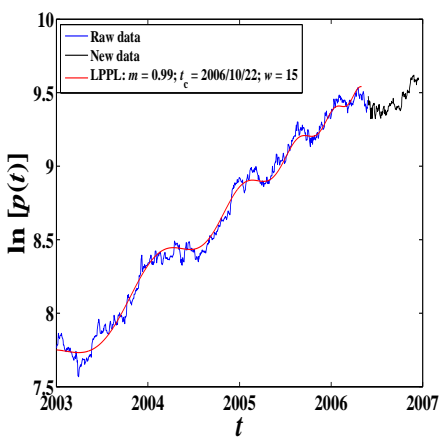

(b) Stock No.29: NPN

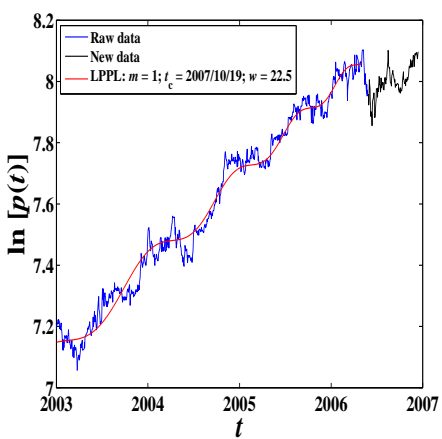

(e) Stock No.32: PIK

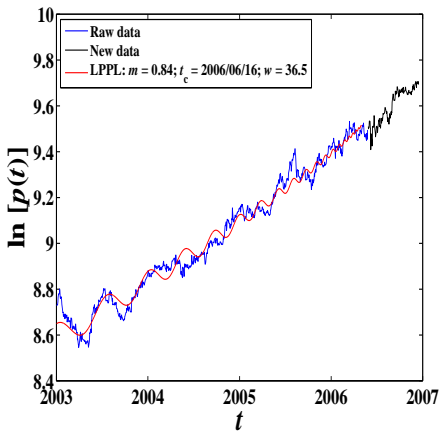

(h) Stock No.35: REM

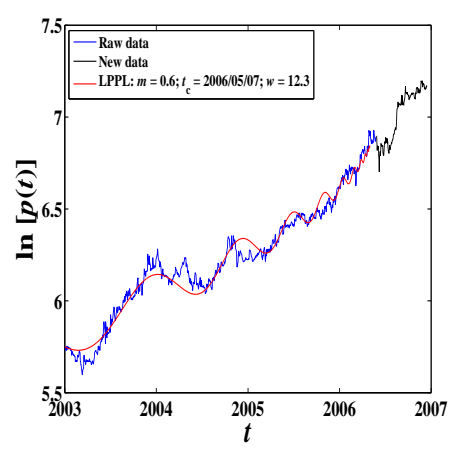

(c) Stock No.30: NTC

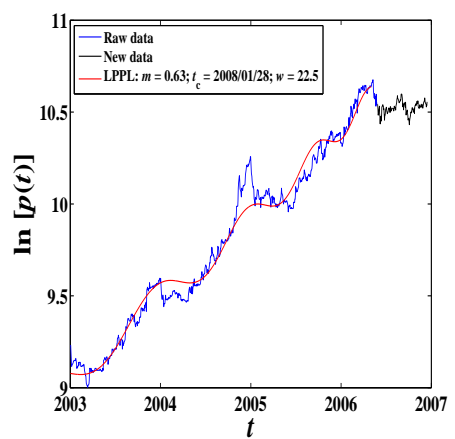

(f) Stock No.33: PPC

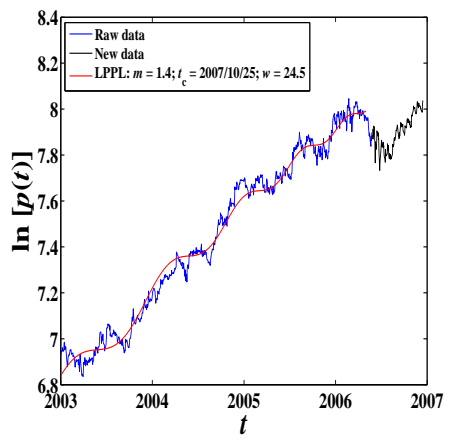

(i) Stock No.36: RMH

Fig. 19. Log-periodic power-law fits of the prices of stocks from No.28 to No.36. 


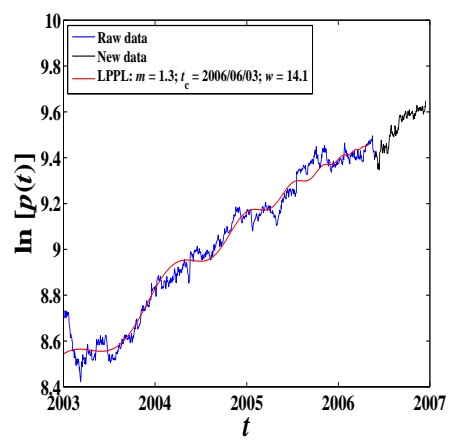

(a) Stock No.37: SAB

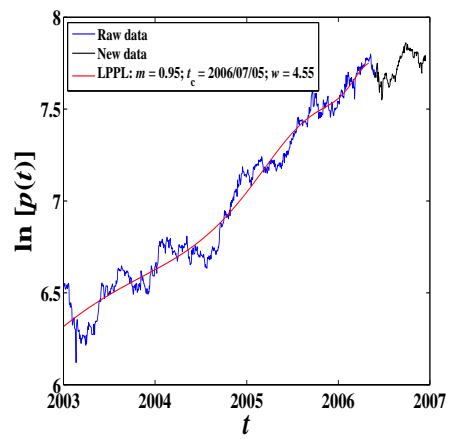

(d) Stock No.40: SHF

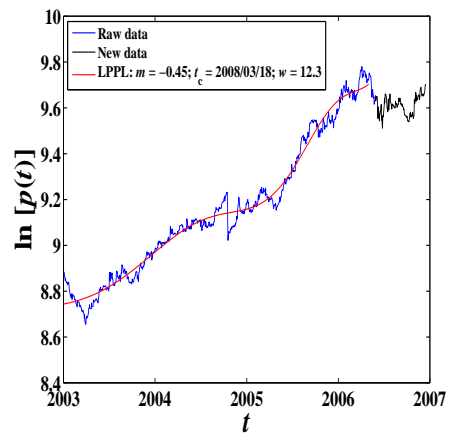

(g) Stock No.43: TBS

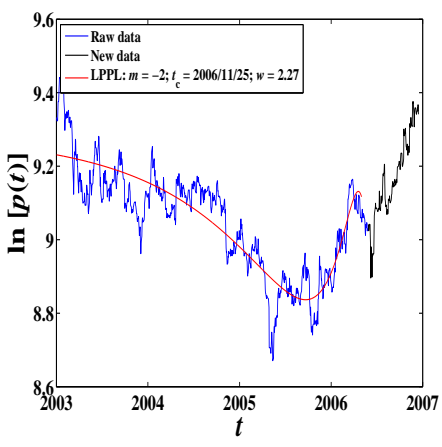

(b) Stock No.38: SAP

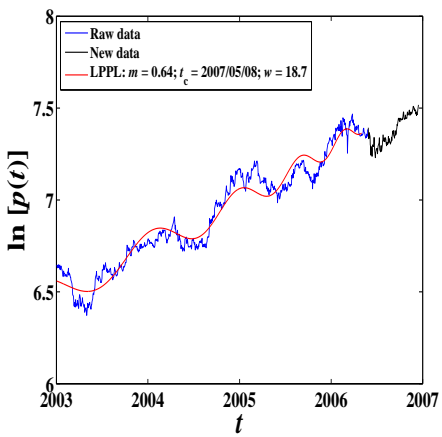

(e) Stock No.41: SLM

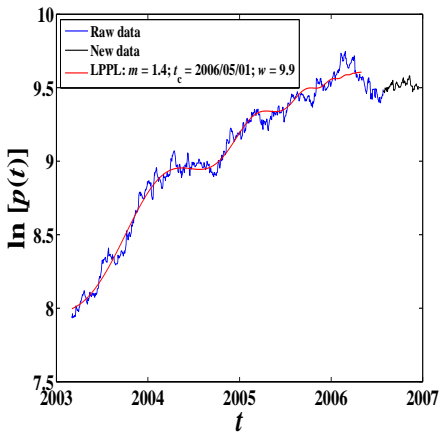

(h) Stock No.44: TKG

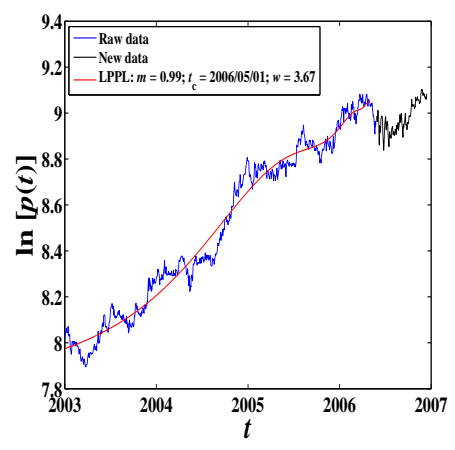

(c) Stock No.39: SBK

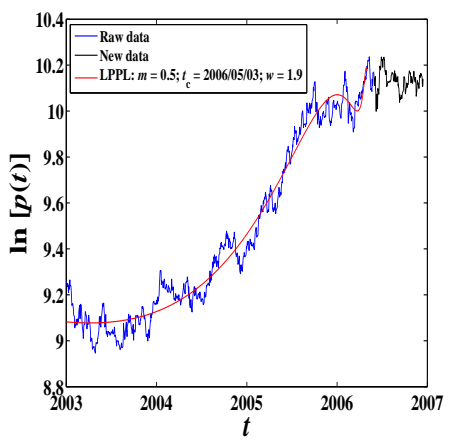

(f) Stock No.42: SOL

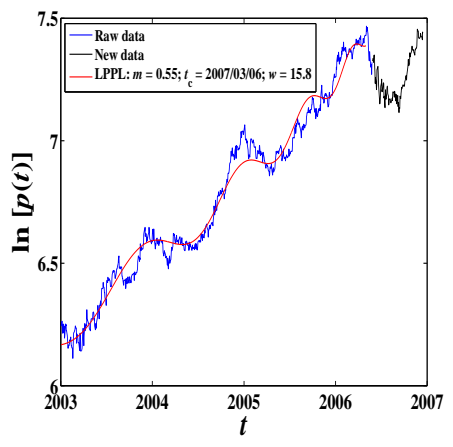

(i) Stock No.45: WHL

Fig. 20. Log-periodic power-law fits of the prices of stocks from No.37 to No.45. 